This is a self-archived version of an original article. This version may differ from the original in pagination and typographic details.

Author(s): Kinnunen, Juha; Lehrbäck, Juha; Vähäkangas, Antti; Zhong, Xiao

Title: Maximal function estimates and self-improvement results for Poincaré inequalities

Year: 2019

Version: Accepted version (Final draft)

Copyright: @ @ Springer-Verlag GmbH Germany, part of Springer Nature 2018

Rights: In Copyright

Rights url: http://rightsstatements.org/page/InC/1.0/?language=en

Please cite the original version:

Kinnunen, J., Lehrbäck, J., Vähäkangas, A., \& Zhong, X. (2019). Maximal function estimates and self-improvement results for Poincaré inequalities. Manuscripta Mathematica, 158(1-2), 119-

147. https://doi.org/10.1007/s00229-018-1016-1 


\title{
MAXIMAL FUNCTION ESTIMATES AND SELF-IMPROVEMENT RESULTS FOR POINCARÉ INEQUALITIES
}

\author{
JUHA KINNUNEN, JUHA LEHRBÄCK, ANTTI V. VÄHÄKANGAS, AND XIAO ZHONG
}

\begin{abstract}
Our main result is an estimate for a sharp maximal function, which implies a KeithZhong type self-improvement property of Poincaré inequalities related to differentiable structures on metric measure spaces. As an application, we give structure independent representation for Sobolev norms and universality results for Sobolev spaces.
\end{abstract}

\section{INTRODUCTION}

Relatively standard assumptions in analysis on metric measure spaces are a doubling condition on the measure and a Poincaré type inequality for a certain class of functions. Roughly speaking a Poincaré inequality transfers infinitesimal information encoded in the derivative to larger scales. It also relates the notion of a derivative to the given measure and, together with the doubling condition, implies Sobolev inequalities. We consider the so-called $\mathcal{D}$-structures introduced in [5], which give a very general notion of a derivative with natural differentiation properties in metric measure spaces. This gives an axiomatic point of view to the theory of Sobolev spaces on metric measure spaces, which includes the standard maximal and upper gradient approaches studied, for example, in [6, 14]. Standard references to analysis on metric measure spaces are $[1,9,10]$.

Keith and Zhong proved in [12] that Poincaré inequalities are self-improving under certain assumptions. More precisely, their result improves a $(1, p)$-Poincaré inequality with $p>1$ to a $(1, p-\varepsilon)$-Poincaré inequality for some $\varepsilon>0$. This open ended property is of fundamental importance not only because of its theoretical interest but also because of its applications, for example, to regularity theory in the calculus of variations, we refer to [12] and references therein. In this work we establish a corresponding self-improvement property for $\mathcal{D}$-structures, see Theorem 5.8 below. Our goal is to give an abstract and transparent argument with a special emphasis on the role of the underlying space and relevant maximal function inequalities. Indeed, instead of a good lambda inequality [12, Proposition 3.1.1], our main result Theorem 4.3 gives a new estimate for the sharp maximal function associated with a given $\mathcal{D}$-structure. This result may be of independent interest and several questions related to weighted norm inequalities for future research arise.

A distinctive feature of our approach is that, in addition to the standard Lipschitz scale, we also consider Hölder continuous functions. Moreover, the role of the underlying space is visible only by way of the $\mathcal{D}$-structure and certain geodesic arguments. On technical level our argument differs from that of [12] in the sense that Whitney type extension theorems for Lipschitz functions are completely avoided and the stopping time argument is tailored for $\mathcal{D}$-stuctures. We would also like to point out that there is only one single place in the proof of Theorem 4.3 where the assumed Poincaré inequality is needed. Another approach to the Keith-Zhong theorem has been recently given in [2].

As an application of our main result we study universality results for Sobolev spaces related to $\mathcal{D}$-structures. More precisely, Theorem 6.2 gives a $\mathcal{D}$-structure independent representation for the Sobolev norm. We also show that any abstract Sobolev space, rising from a suitable $\mathcal{D}$ structure, is isomorphic to one particular Sobolev space. This extends and complements results in $[14,15]$.

Date: May 15, 2017.

2010 Mathematics Subject Classification. 42B25, 35A23, 46E35, 31E05, 30L99.

Key words and phrases. Analysis on metric spaces, Sobolev spaces, Poincaré inequality, geodesic space.

The research is supported by the Academy of Finland. 


\section{Preliminaries}

\subsection{Tracking constants}

Our results are based on quantitative estimates and absorption arguments, where it is often crucial to track the dependencies of constants quantitatively. For this purpose, we will use the following notational convention: $C(*, \cdots, *)$ denotes a positive constant which quantitatively depends on the quantities indicated by the $*$ 's but whose actual value can change from one occurrence to another, even within a single line.

\subsection{Metric spaces}

Here, and throughout the paper, we assume that $X=(X, d, \mu)$ is a metric measure space equipped with a metric $d$ and a positive complete Borel measure $\mu$ such that $0<\mu(B)<\infty$ for all balls $B \subset X$, each of which is always an open set of the form

$$
B=B(x, r)=\{y \in X: d(y, x)<r\}
$$

with $x \in X$ and $r>0$. As in [1, p. 2], we extend $\mu$ as a Borel regular (outer) measure on $X$. We remark that the space $X$ is separable under these assumptions, see [1, Proposition 1.6]. We also assume that $\# X \geq 2$ and that the measure $\mu$ is doubling, that is, there is a constant $c_{\mu}>1$, called the doubling constant of $\mu$, such that

$$
\mu(2 B) \leq c_{\mu} \mu(B)
$$

for all balls $B=B(x, r)$ in $X$. Here we use for $0<t<\infty$ the notation $t B=B(x, \operatorname{tr})$. In particular, for all balls $B=B(x, r)$ that are centered at $x \in A \subset X$ with radius $r \leq \operatorname{diam}(A)$, we have that

$$
\frac{\mu(B)}{\mu(A)} \geq 2^{-s}\left(\frac{r}{\operatorname{diam}(A)}\right)^{s}
$$

where $s=\log _{2} c_{\mu}>0$. We refer to [9, p. 31].

\subsection{Geodesic spaces}

Let $X$ be a metric space satisfying the conditions stated in $§ 2.2$. By a curve we mean a nonconstant, rectifiable, continuous mapping from a compact interval of $\mathbb{R}$ to $X$; we tacitly assume that all curves are parametrized by their arc-length. We say that $X$ is a geodesic space, if every pair of points in $X$ can be joined by a curve whose length is equal to the distance between the two points. In particular, it easily follows that

$$
0<\operatorname{diam}(2 B) \leq 4 \operatorname{diam}(B)
$$

for all balls $B=B(x, r)$ in a geodesic space $X$.

The following lemma is [10, Lemma 12.1.2].

Lemma 2.4. Suppose that $X$ is a geodesic space and $A \subset X$ is a measurable set. Then the function

is continuous whenever $x \in X$.

$$
r \mapsto \frac{\mu(B(x, r) \cap A)}{\mu(B(x, r))}:(0, \infty) \rightarrow \mathbb{R}
$$

Lemma 2.5. Suppose that $B=B(x, r)$ and $B^{\prime}=B\left(x^{\prime}, r^{\prime}\right)$ are two balls in a geodesic space $X$ such that $x^{\prime} \in B$ and $0<r^{\prime} \leq \operatorname{diam}(B)$. Then $\mu\left(B^{\prime}\right) \leq c_{\mu}^{3} \mu\left(B^{\prime} \cap B\right)$.

Proof. It suffices to find $y \in X$ such that $B\left(y, r^{\prime} / 4\right) \subset B^{\prime} \cap B$. Inequality $\mu\left(B^{\prime}\right) \leq c_{\mu}^{3} \mu\left(B^{\prime} \cap B\right)$ then follows from the doubling condition (2.1) and the fact that $B^{\prime} \subset B\left(y, 2 r^{\prime}\right)$.

Assume first that $x \in B\left(x^{\prime}, r^{\prime} / 4\right)$. In this case we may choose $y=x^{\prime}$, since we have for all $z \in B\left(x^{\prime}, r^{\prime} / 4\right)$ that

$$
d(z, x) \leq d\left(z, x^{\prime}\right)+d\left(x^{\prime}, x\right)<r^{\prime} / 4+r^{\prime} / 4=r^{\prime} / 2 \leq \operatorname{diam}(B) / 2 \leq r,
$$

and hence $B\left(x^{\prime}, r^{\prime} / 4\right) \subset B^{\prime} \cap B(x, r)=B^{\prime} \cap B$. 
Let us then consider the case $x \notin B\left(x^{\prime}, r^{\prime} / 4\right)$. Since $X$ is a geodesic space, there exists an arc-length parametrized curve $\gamma:[0, \ell] \rightarrow X$ with $\gamma(0)=x^{\prime}, \gamma(\ell)=x$ and $\ell=d\left(x, x^{\prime}\right)$. We claim that $y=\gamma\left(r^{\prime} / 4\right)$ satisfies the required condition $B\left(y, r^{\prime} / 4\right) \subset B^{\prime} \cap B$. In order to prove the claim, let us fix a point $z \in B\left(y, r^{\prime} / 4\right)$. Then

$$
d\left(z, x^{\prime}\right) \leq d(z, y)+d\left(y, x^{\prime}\right)<r^{\prime} / 4+d\left(\gamma\left(r^{\prime} / 4\right), \gamma(0)\right) \leq r^{\prime} / 2<r^{\prime} .
$$

Hence $z \in B\left(x^{\prime}, r^{\prime}\right)$ and therefore $B\left(y, r^{\prime} / 4\right) \subset B\left(x^{\prime}, r^{\prime}\right)=B^{\prime}$. Moreover, since $\ell=d\left(x, x^{\prime}\right)$,

$$
\begin{aligned}
d(z, x) & \leq d(z, y)+d(y, x)<r^{\prime} / 4+d\left(\gamma\left(r^{\prime} / 4\right), \gamma(\ell)\right) \\
& \leq r^{\prime} / 4+\left(\ell-r^{\prime} / 4\right)=\ell=d\left(x, x^{\prime}\right)<r .
\end{aligned}
$$

It follows that $z \in B(x, r)=B$ and therefore $B\left(y, r^{\prime} / 4\right) \subset B^{\prime} \cap B$.

\subsection{Hölder and Lipschitz functions}

Let $A \subset X$. We say that $u: A \rightarrow \mathbb{R}$ is a $\beta$-Hölder function, with an exponent $0<\beta \leq 1$ and a constant $0 \leq \kappa<\infty$, if

$$
|u(x)-u(y)| \leq \kappa d(x, y)^{\beta} \quad \text { for all } x, y \in A .
$$

If $u: A \rightarrow \mathbb{R}$ is a $\beta$-Hölder function, with a constant $\kappa$, then the classical McShane extension

$$
v(x)=\inf \left\{u(y)+\kappa d(x, y)^{\beta}: y \in A\right\}, \quad x \in X,
$$

defines a $\beta$-Hölder function $v: X \rightarrow \mathbb{R}$, with the constant $\kappa$, which satisfies $\left.v\right|_{A}=u$; we refer to [9, pp. 43-44]. The set of all $\beta$-Hölder functions $u: A \rightarrow \mathbb{R}$ is denoted by $\operatorname{Lip}_{\beta}(A)$. The 1-Hölder functions are also called Lipschitz functions. We denote $\operatorname{Lip}(A)=\operatorname{Lip}_{1}(A)$.

\section{Definition and basic properties of $\mathcal{D}$-STRuCtures}

We adapt the terminology from [5] concerning the so-called $\mathcal{D}$-structures. This structural framework captures the properties that we will need for Keith-Zhong type self-improvement of Poincare inequalities, treated in $\S 4-\S 6$. In the following definition, and throughout the paper, we use the following familiar notation:

$$
u_{A}=f_{A} u(y) d \mu(y)=\frac{1}{\mu(A)} \int_{A} u(y) d \mu(y)
$$

is the integral average of $u \in L^{1}(A)$ over a measurable set $A \subset X$ with $0<\mu(A)<\infty$. Moreover if $E \subset X$, then $\mathbf{1}_{E}$ denotes the characteristic function of $E$; that is, $\mathbf{1}_{E}(x)=1$ if $x \in E$ and $\mathbf{1}_{E}(x)=0$ if $x \in X \backslash E$.

Definition 3.1. Let $X$ be a metric measure space (recall $\S 2.2$ ). Fix $1 \leq p<\infty$ and $0<\beta \leq 1$. Suppose that for each $u \in \operatorname{Lip}_{\beta}(X)$, we are given a family $\mathcal{D}(u) \neq \emptyset$ of measurable functions $X \rightarrow[0, \infty]$ as follows. First, we assume the following Poincaré inequality condition:

(D1) There are constants $K>0$ and $\tau \geq 1$ such that the $(1, p)$-Poincaré inequality

$$
f_{B}\left|u(x)-u_{B}\right| d \mu(x) \leq K^{1 / p} \operatorname{diam}(B)^{\beta}\left(f_{\tau B} g(x)^{p} d \mu(x)\right)^{1 / p}
$$

holds whenever $B$ is a ball in $X$ and whenever $u \in \operatorname{Lip}_{\beta}(X)$ and $g \in \mathcal{D}(u)$.

Second, for all $\beta$-Hölder functions $u, v: X \rightarrow \mathbb{R}$, we assume the following conditions (D2)-(D4):

(D2) $|a| g \in \mathcal{D}(a u)$ if $a \in \mathbb{R}$ and $g \in \mathcal{D}(u)$;

(D3) $g_{u}+g_{v} \in \mathcal{D}(u+v)$ if $g_{u} \in \mathcal{D}(u)$ and $g_{v} \in \mathcal{D}(v)$;

(D4) If $v: X \rightarrow \mathbb{R}$ is $\beta$-Hölder with a constant $\kappa \geq 0$ and $\left.v\right|_{X \backslash E}=\left.u\right|_{X \backslash E}$ for a Borel set $E \subset X$, then $\kappa \mathbf{1}_{E}+g_{u} \mathbf{1}_{X \backslash E} \in \mathcal{D}(v)$ whenever $g_{u} \in \mathcal{D}(u)$.

Then we say that the family $\left\{\mathcal{D}(u): u \in \operatorname{Lip}_{\beta}(X)\right\}$ is a $\mathcal{D}$-structure in $X$, with exponents $1 \leq p<\infty$ and $0<\beta \leq 1$, and with constants $K>0$ and $\tau \geq 1$.

Later in $\S 4$ we will need a stronger form of the condition (D1). This stronger form (D1'), corresponding to a $(p, p)$-Poincaré inequality, is explicitly stated in the following theorem. 
Theorem 3.3. Suppose that $\left\{\mathcal{D}(u): u \in \operatorname{Lip}_{\beta}(X)\right\}$ is a $\mathcal{D}$-structure in a geodesic space $X$, with exponents $1 \leq p<\infty$ and $0<\beta \leq 1$, and with constants $K>0$ and $\tau \geq 1$. Then the following condition is valid:

(D1') There exists $K_{p, p}=C\left(c_{\mu}, \beta, p, q, \tau\right) K>0$ such that the $(p, p)$-Poincaré inequality

$$
\left(f_{B}\left|u(x)-u_{B}\right|^{p} d \mu(x)\right)^{1 / p} \leq K_{p, p}^{1 / p} \operatorname{diam}(B)^{\beta}\left(f_{B} g(x)^{p} d \mu(x)\right)^{1 / p}
$$

holds whenever $B$ is a ball in $X$ and whenever $u \in \operatorname{Lip}_{\beta}(X)$ and $g \in \mathcal{D}(u)$.

Theorem 3.3 is an immediate consequence of a stronger result, namely the $\mathcal{D}$-structure independent Theorem 3.6. Moreover, the latter result gives $(q, p)$-Poincaré inequalities for some $q>p$. By formulating Theorem 3.6 separately, we wish to emphasize the contrast that $\mathcal{D}$ structures are not needed in this 'simpler' aspect of self-improvement.

We need a chaining lemma from [9, p. 30-31].

Lemma 3.4. Suppose that $X$ is a geodesic space and that $\tau \geq 1$. Then there are constants $M=C(\tau) \geq 1$ and $a=C(\tau)>1$ as follows.

Every ball $B \subset X$ contains a ball $B_{0} \subset B$ such that, for each $x \in B$, there is a sequence of balls $\left\{B_{i}: i=1,2, \ldots\right\}$ in $X$ satisfying the following conditions:

(a) $\tau B_{i} \subset B$ for all $i \geq 0$;

(b) $B_{i}$ is centered at $x$ for all sufficiently large $i$;

(c) the radius $r_{i}$ of $B_{i}$ satisfies $M^{-1} a^{-i} \operatorname{diam}(B) \leq r_{i} \leq M a^{-i} \operatorname{diam}(B)$ for all $i \geq 0$; and

(d) the intersection $B_{i} \cap B_{i+1}$ contains a ball $R_{i}$ such that $B_{i} \cup B_{i+1} \subset M R_{i}$ for all $i \geq 0$.

We also need the following lemma, which is essentially [9, Lemma 4.22]. See also [3, p. 485].

Lemma 3.5. Let $B \subset X$ be a ball in a metric space and let $u: B \rightarrow \mathbb{R}$ be a measurable function. Fix $1 \leq q<t<\infty$ and $C_{0}>0$ such that

$$
\mu(\{x \in B:|u(x)|>\lambda\}) \leq C_{0} \lambda^{-t}
$$

for each $\lambda>0$. Then

$$
\left(f_{B}|u|^{q} d \mu\right)^{1 / q} \leq 2^{1 / q}\left(\frac{C_{0} q}{t-q}\right)^{1 / t} \mu(B)^{-1 / t} .
$$

The following self-improvement result follows from a straightforward adaptation of the main result in [8] that corresponds to the case $\beta=1$. We refer to [4] for versions of this result taking place in general metric spaces and with any $\beta>0$. For convenience, we recall the proof.

Theorem 3.6. Suppose that $X$ is a geodesic space. Fix exponents $1 \leq p<\infty$ and $0<\beta \leq 1$. Suppose that $u \in \operatorname{Lip}_{\beta}(X)$ and that $g: X \rightarrow[0, \infty]$ is a measurable function. Assume further that there are constants $K>0$ and $\tau \geq 1$ such that inequality

$$
f_{B}\left|u(x)-u_{B}\right| d \mu(x) \leq K^{1 / p} \operatorname{diam}(B)^{\beta}\left(f_{\tau B} g(x)^{p} d \mu(x)\right)^{1 / p}
$$

holds whenever $B$ is a ball in $X$. Suppose that $Q \geq \log _{2} c_{\mu}>0$ satisfies inequality $\beta p<Q$, where $c_{\mu}$ is the doubling constant of $\mu$. Fix $1 \leq q<Q p /(Q-\beta p)$. Then there is a constant $C=C\left(c_{\mu}, Q, \beta, p, q, \tau\right)>0$ such that inequality

$$
\left(f_{B}\left|u(x)-u_{B}\right|^{q} d \mu(x)\right)^{1 / q} \leq C K^{1 / p} \operatorname{diam}(B)^{\beta}\left(f_{B} g(x)^{p} d \mu(x)\right)^{1 / p}
$$

holds whenever $B \subset X$ is a ball.

Proof. Fix $u, g$ and a ball $B=B\left(x_{0}, r\right) \subset X$ with $r>0$. Without loss of generality, we may assume that $r \leq 2 \operatorname{diam}(B)$. Let $B_{0} \subset B$ be the fixed ball as in Lemma 3.4 for the given $B \subset X$ and $\tau \geq 1$. By subtracting a constant from $u$, if necessary, we can assume that $u_{B_{0}}=0$. 
Let $\lambda>0$ and let $x \in U^{\lambda}=\{y \in B:|u(y)|>\lambda\}$. Fix $\left\{B_{i}=B\left(x_{i}, r_{i}\right): i=1,2, \ldots\right\}$ and $\left\{R_{i}: i=0,1, \ldots\right\}$ that are associated with the point $x$ and the ball $B$ as in Lemma 3.4. In particular, the properties (a)-(d) of the chain are valid. By the properties (b) and (c), we have $u_{B_{i}} \rightarrow u(x)$ as $i \rightarrow \infty$, and so

$$
\begin{aligned}
\lambda & <|u(x)|=\left|u(x)-u_{B_{0}}\right| \leq \sum_{i=0}^{\infty}\left|u_{B_{i+1}}-u_{B_{i}}\right| \\
& \leq \sum_{i=0}^{\infty}\left(\left|u_{B_{i+1}}-u_{R_{i}}\right|+\left|u_{R_{i}}-u_{B_{i}}\right|\right) \\
& \leq \sum_{i=0}^{\infty}\left(\frac{\mu\left(B_{i+1}\right)}{\mu\left(R_{i}\right)} f_{B_{i+1}}\left|u-u_{B_{i+1}}\right| d \mu+\frac{\mu\left(B_{i}\right)}{\mu\left(R_{i}\right)} f_{B_{i}}\left|u-u_{B_{i}}\right| d \mu\right) \\
& \leq C K^{1 / p} \sum_{i=0}^{\infty} r_{i}^{\beta}\left(f_{\tau B_{i}} g^{p} d \mu\right)^{1 / p} .
\end{aligned}
$$

Hence for any $0<\varepsilon<1$, that is to be chosen later, we obtain that

$$
\sum_{i=0}^{\infty} \lambda r^{-\beta \varepsilon} r_{i}^{\beta \varepsilon} \leq C \lambda r^{-\beta \varepsilon} \sum_{i=0}^{\infty}\left(a^{-i} \operatorname{diam}(B)\right)^{\beta \varepsilon} \leq C \lambda \leq C K^{1 / p} \sum_{i=0}^{\infty} r_{i}^{\beta}\left(f_{\tau B_{i}} g^{p} d \mu\right)^{1 / p} .
$$

By comparing the sums on the left and right, we obtain an index $i_{x} \in\{0,1, \ldots\}$ such that

$$
\lambda r^{-\beta \varepsilon} r_{i_{x}}^{\beta \varepsilon} \leq C K^{1 / p} r_{i_{x}}^{\beta}\left(f_{\tau B_{i_{x}}} g^{p} d \mu\right)^{1 / p}
$$

A straightforward chaining argument, relying on the properties (b)-(d) of the chain, implies that $x \in C(M, a) B_{i_{x}}=B_{i_{x}}^{\prime}$ for a constant $C(M, a) \geq 1$. By the previous estimates and property (a) of the chain,

$$
\lambda^{p} r_{i_{x}}^{\beta p(\varepsilon-1)} \mu\left(B_{i_{x}}\right) \leq C K r^{\beta p \varepsilon} \int_{\tau B_{i_{x}}} g^{p} d \mu \leq C K r^{\beta p \varepsilon} \int_{\tau B_{i_{x}}^{\prime}} \mathbf{1}_{B} g^{p} d \mu .
$$

The assumptions on $Q$, inequality (2.2), and properties (a) and (c) together imply that

$$
\frac{\mu\left(B_{i_{x}}\right)}{\mu(B)} \geq \frac{\mu\left(M^{-1} B_{i_{x}}\right)}{\mu(B)} \geq C\left(\frac{r_{i_{x}}}{\operatorname{diam}(B)}\right)^{Q} \geq C\left(\frac{r_{i_{x}}}{r}\right)^{Q} .
$$

By first raising this to power $\beta p(\varepsilon-1) / Q<0$ and then substituting the result to (3.7),

$$
\begin{aligned}
\lambda^{p} \mu\left(5 \tau B_{i_{x}}^{\prime}\right)^{1+\beta p(\varepsilon-1) / Q} & \leq C \lambda^{p} \mu\left(B_{i_{x}}\right)^{1+\beta p(\varepsilon-1) / Q} \\
& \leq C K r^{\beta p} \mu(B)^{\beta p(\varepsilon-1) / Q} \int_{\tau B_{i_{x}}^{\prime}} \mathbf{1}_{B} g^{p} d \mu
\end{aligned}
$$

Using the $5 r$-covering lemma [1, Lemma 1.7], we obtain a countable and disjoint subfamily

$$
\left\{\tau B_{x_{k}}^{\prime}\right\} \subset\left\{\tau B_{i_{x}}^{\prime}: x \in U^{\lambda}\right\}
$$

of balls indexed by $k$ such that the covering property $U^{\lambda} \subset \cup_{k} 5 \tau B_{x_{k}}^{\prime}$ holds true. Let us also observe that $0<1+\beta p(\varepsilon-1) / Q<1$. Hence, by the above covering property and (3.8),

$$
\begin{aligned}
\lambda^{p} \mu\left(U^{\lambda}\right)^{1+\beta p(\varepsilon-1) / Q} & \leq \sum_{k} \lambda^{p} \mu\left(5 \tau B_{x_{k}}^{\prime}\right)^{1+\beta p(\varepsilon-1) / Q} \\
& \leq C K r^{\beta p} \mu(B)^{\beta p(\varepsilon-1) / Q} \sum_{k} \int_{\tau B_{x_{k}}^{\prime}} \mathbf{1}_{B} g^{p} d \mu \\
& \leq C K r^{\beta p} \mu(B)^{\beta p(\varepsilon-1) / Q} \int_{B} g^{p} d \mu
\end{aligned}
$$


Recall that $\beta p<Q$ and $1 \leq q<Q p /(Q-\beta p)$. These facts allows us to choose the number $0<\varepsilon<1$, depending on $Q, p$ and $\beta$ only, such that $\max \{q, p\}<t=p /(1+\beta p(\varepsilon-1) / Q)$. Thus, by raising inequality (3.9) to the power $t / p$ and applying Lemma 3.5, we obtain

$$
\begin{aligned}
\left(f_{B}\left|u-u_{B}\right|^{q} d \mu\right)^{t / q} & \leq 2^{t}\left(f_{B}|u|^{q} d \mu\right)^{t / q} \\
& \leq \frac{C \sup _{\lambda>0} \lambda^{t} \mu(\{x \in B:|u(x)|>\lambda\})}{\mu(B)} \\
& \leq C K^{t / p} r^{\beta t}\left(f_{B} g^{p} d \mu\right)^{t / p} .
\end{aligned}
$$

Since $B \subset X$ is an arbitrary ball, we conclude the proof by raising both sides to power $1 / t$ and recalling that $r \leq 2 \operatorname{diam}(B)$.

\section{BOUNDEDNESS RESUlTS FOR MAXIMAL OPERATORS}

\subsection{The main result}

Here we formulate and prove our main result, Theorem 4.3. This theorem can be viewed as a boundedness result for a certain maximal function which, in turn, is naturally associated with a given $\mathcal{D}$-structure. More specifically, let $1<p<\infty$ and $0<\beta \leq 1$. If $\mathcal{B} \neq \emptyset$ is a given family of balls in $X$, then we define a fractional sharp maximal function

$$
M_{\beta, \mathcal{B}}^{\sharp, p} u(x)=\sup _{x \in B \in \mathcal{B}}\left(\frac{1}{\operatorname{diam}(B)^{\beta p}} f_{B}\left|u(y)-u_{B}\right|^{p} d \mu(y)\right)^{1 / p}, \quad x \in X,
$$

whenever $u: X \rightarrow \mathbb{R}$ is a $\beta$-Hölder function. The supremum above is defined to be zero, if there is no ball $B$ in $\mathcal{B}$ that contains the point $x$.

We are primarily interested in the localized maximal function $M_{\beta, \mathcal{B}_{0}}^{\sharp, p} u$ that is associated with the ball family

$$
\mathcal{B}_{0}=\left\{B \subset X: B \text { is a ball such that } 2 B \subset B_{0}\right\} ;
$$

here and in the statement of Theorem 4.3, the set $B_{0} \subset X$ of localization is a fixed ball, and the case $X=B_{0}$ is allowed but then $X$ is of course necessarily bounded.

Theorem 4.3. Suppose we are given a $\mathcal{D}$-structure in a geodesic space $X$, with exponents $1<p<\infty$ and $0<\beta \leq 1$. Let $K_{p, p}>0$ be the constant for the $(p, p)$-Poincaré inequality as in condition (D1') of Theorem 3.3. Let $k \in \mathbb{N}, 0 \leq \varepsilon<p-1$, and $\alpha=\beta p^{2} /(2(s+\beta p))>0$ with $s=\log _{2} c_{\mu}$. Suppose that $B_{0} \subset X$ is a fixed ball. Then inequality

$$
\begin{aligned}
\int_{B_{0}}\left(M_{\beta, \mathcal{B}_{0}}^{\sharp, p} u\right)^{p-\varepsilon} d \mu \leq & C_{1}\left(2^{k(\varepsilon-\alpha)}+\frac{K_{p, p} 4^{k \varepsilon}}{k^{p-1}}\right) \int_{B_{0}}\left(M_{\beta, \mathcal{B}_{0}}^{\sharp, p} u\right)^{p-\varepsilon} d \mu \\
& +C_{1} C(k, \varepsilon) K_{p, p} \int_{B_{0} \backslash\left\{M_{\beta, \mathcal{B}_{0}}^{\sharp, p} u=0\right\}} g^{p}\left(M_{\beta, \mathcal{B}_{0}}^{\sharp, p} u\right)^{-\varepsilon} d \mu
\end{aligned}
$$

holds for each $u \in \operatorname{Lip}_{\beta}(X)$ and every $g \in \mathcal{D}(u)$. Here the constant $C_{1}>0$ depends only on the parameters $\beta, p, c_{\mu}$; and $C(k, \varepsilon)=\left(4^{k \varepsilon}-1\right) / \varepsilon$ if $\varepsilon>0$ and $C(k, 0)=k$.

Let us observe that the first term on the right-hand side of (4.4) is finite, since $u$ is assumed to be a $\beta$-Hölder function. The following corollary is obtained when this term is absorbed to the left-hand side after choosing the numbers $k$ and $0 \leq \varepsilon<\varepsilon_{0}$ appropriately; for instance, we can choose $\varepsilon_{0}=1 / k$ for a large enough $k$.

Corollary 4.5. Suppose that we are given a $\mathcal{D}$-structure in a geodesic space $X$, with exponents $1<p<\infty$ and $0<\beta \leq 1$. Then there exists some $0<\varepsilon_{0}<p-1$ with the property that for every $0 \leq \varepsilon<\varepsilon_{0}$ there is a constant $C>0$ such that inequality

$$
\int_{B_{0}}\left(M_{\beta, \mathcal{B}_{0}}^{\sharp, p} u\right)^{p-\varepsilon} d \mu \leq C \int_{B_{0} \backslash\left\{M_{\beta, \mathcal{B}_{0}}^{\sharp, p} u=0\right\}} g^{p}\left(M_{\beta, \mathcal{B}_{0}}^{\sharp, p} u\right)^{-\varepsilon} d \mu
$$


holds whenever $B_{0}$ is a ball in $X$ and whenever $u \in \operatorname{Lip}_{\beta}(X)$ and $g \in \mathcal{D}(u)$.

Question 4.7. Corollary 4.5 suggests the following problem related to weighted inequalities. Fix $1<p<\infty$ and $0<\beta \leq 1$. Let us denote by $\mathcal{B}$ the family of all balls in $X$. Then, for some interesting $\mathcal{D}$-structure, is it possible to characterize those weights $w$ in $X$ for which inequality

$$
\int_{X}\left(M_{\beta, \mathcal{B}}^{\sharp, p} u\right)^{p} w d \mu \leq C \int_{X} g^{p} w d \mu
$$

holds for each $u \in \operatorname{Lip}_{\beta}(X)$ and for every $g \in \mathcal{D}(u)$ ? To our knowledge, this is an open problem even when $X=\mathbb{R}^{n}$ equipped with the Lebesgue measure.

Remark 4.8. It is instructive to reflect Question 4.7 and Corollary 4.5 by considering the following simple analogy with $X=\mathbb{R}^{n}$ equipped with the Lebesgue measure. If $1<p<\infty$, then the Muckenhoupt $A_{p}$ class consists precisely of weights $w$ for which the maximal operator

$$
u \mapsto M u=\sup \left\{|u|_{B} \mathbf{1}_{B}: B \subset \mathbb{R}^{n} \text { is any ball }\right\}
$$

is bounded on $L^{p}(w d x)$. Whereas Question 4.7 asks for a counterpart of this classical result in the present setting, Corollary 4.5, in turn, corresponds to a rather curious special case. Namely, let $0 \leq \delta<1$ and let $u$ be a measurable function with $0<\|u\|_{\infty}<\infty$. Then $(M u)^{\delta}$ is a Muckenhoupt $A_{1}$ weight whose $A_{1}$-constant is independent of $u$; cf. [3, Theorem 3.4 in $\S 2$ ]. As a consequence, the function $w=(M u)^{-\varepsilon}$ is an $A_{p}$ weight if $\varepsilon=\delta(p-1)>0$. Moreover, the $A_{p}$ constant of this weight is independent of $u$. By the boundedness of the maximal function in $L^{p}(w d x)$, and the fact that $w(x) \leq|u(x)|^{-\varepsilon}$ almost everywhere, we find that

$$
\begin{aligned}
\int_{\mathbb{R}^{n}}(M u(x))^{p-\varepsilon} d x & =\int_{\mathbb{R}^{n}}(M u(x))^{p} w(x) d x \\
& \leq C \int_{\mathbb{R}^{n}}|u(x)|^{p} w(x) d x \leq C \int_{\mathbb{R}^{n}}|u(x)|^{p-\varepsilon} d x .
\end{aligned}
$$

In some cases, see $\S 5$ in particular, we can further adapt this computation to the present setting.

The proof of Theorem 4.3 is completed in $\S 4.6$. For the proof, we need preparations that are treated in $\S 4.2-\S 4.5$. At this stage, we already fix $X$, the $\mathcal{D}$-structure, $K_{p, p}, B_{0} \subsetneq X, \mathcal{B}_{0}, p, \beta$, $\varepsilon, k$ and $u$ as in the statement of Theorem 4.3. We refer to these objects throughout $\S 4$ without further notice. Notice, however, that the function $g$ is not yet fixed.

Let us emphasize that the ball $B_{0}$ in the proof below is further assumed to be a strict subset of $X$. That is, we will only focus on the case $B_{0} \neq X$. We remark that if $B_{0}=X$, then $X$ is bounded and the following Whitney cover $\mathcal{W}_{0}$ is replaced with the singleton $\left\{Q=B_{0}\right\}$. The other modifications in this easier special case are straightforward and we omit the details.

\subsection{Whitney ball covering}

We need a Whitney ball covering $\mathcal{W}_{0}=\mathcal{W}\left(B_{0}\right)$ of the ball $B_{0} \subsetneq X$. This countable family with good covering properties is comprised of the so-called Whitney balls that are of the form $Q=B\left(x_{Q}, r_{Q}\right) \in \mathcal{W}_{0}$, with center $x_{Q} \in B_{0}$ and radius

$$
r_{Q}=\frac{\operatorname{dist}\left(x_{Q}, X \backslash B_{0}\right)}{128}>0 .
$$

The 4-dilated Whitney ball is denoted by $Q^{*}=4 Q=B\left(x_{Q}, 4 r_{Q}\right)$ whenever $Q \in \mathcal{W}_{0}$. Even though the Whitney balls need not be pairwise disjoint, they nevertheless have the following standard covering properties with bounded overlap; cf. [1, pp. 77-78].

(W1) $B_{0}=\bigcup_{Q \in \mathcal{W}_{0}} Q$;

(W2) $\sum_{Q \in \mathcal{W}_{0}} \mathbf{1}_{Q^{*}} \leq C \mathbf{1}_{B_{0}}$ for some constant $C=C\left(c_{\mu}\right)>0$.

The facts (W3)-(W6) below for any Whitney ball $Q=B\left(x_{Q}, r_{Q}\right) \in \mathcal{W}_{0}$ are straightforward to verify by using inequality (2.3) and the assumption $B_{0} \subsetneq X$; we omit the simple proofs. Below we refer to the family $\mathcal{B}_{0}$ of balls that is defined in (4.2) by using the fixed ball $B_{0}$.

(W3) If $B \subset X$ is a ball such that $B \cap Q \neq \emptyset \neq 2 B \cap\left(X \backslash Q^{*}\right)$, then $\operatorname{diam}(B) \geq 3 r_{Q} / 4$; 
(W4) If $B \subset Q^{*}$ is a ball, then $B \in \mathcal{B}_{0}$;

(W5) If $B \subset Q^{*}$ is a ball, $x \in B$ and $0<r \leq \operatorname{diam}(B)$, then $B(x, 5 r) \in \mathcal{B}_{0}$;

(W6) If $x \in Q^{*}$ and $0<r \leq 2 \operatorname{diam}\left(Q^{*}\right)$, then $B(x, r) \in \mathcal{B}_{0}$.

Observe that there is some overlap between the properties (W4)-(W6). The slightly different formulations will conveniently guide the reader in the sequel.

\subsection{Fractional sharp maximal functions}

We abbreviate $M^{\sharp} u=M_{\beta, \mathcal{B}_{0}}^{\sharp} u$ and denote

$$
U^{\lambda}=\left\{x \in B_{0}: M^{\sharp} u(x)>\lambda\right\}, \quad \lambda>0 .
$$

The sets $U^{\lambda}$ are open in $X$. If $E \subset X$ is a Borel set and $\lambda>0$, we write $U_{E}^{\lambda}=U^{\lambda} \cap E$. We also need a certain smaller maximal function that is localized to Whitney balls. More specifically, for each $Q \in \mathcal{W}_{0}$, we first consider the ball family ${ }^{1}$

$$
\mathcal{B}_{Q}=\left\{B \subset X: B \text { is a ball such that } B \subset Q^{*}\right\}
$$

and define $M_{Q}^{\sharp} u=M_{\beta, \mathcal{B}_{Q}}^{\sharp, p} u$. By using these individual maximal functions, we then define a Whitney-ball localized sharp maximal function ${ }^{2}$

$$
M_{\mathrm{loc}}^{\sharp} u=\sup _{Q \in \mathcal{W}_{0}} \mathbf{1}_{Q} M_{Q}^{\sharp} u .
$$

If $\lambda>0$ and $Q \in \mathcal{W}_{0}$, we write

$$
Q^{\lambda}=\left\{x \in Q: M_{Q}^{\sharp} u(x)>\lambda\right\} \quad \text { and } \quad V^{\lambda}=\left\{x \in B_{0}: M_{\mathrm{loc}}^{\sharp} u(x)>\lambda\right\} .
$$

We need the following norm estimate between the different maximal functions. Its purpose, roughly speaking, is to create space for the forthcoming stopping balls in $\S 4.4$ to expand, without losing their control in terms of $M^{\sharp} u$. On the other hand, controlling this expansion is the only purpose for introducing the different maximal functions aside from $M^{\sharp} u$.

Lemma 4.10. There is a constant $C=C\left(c_{\mu}, p, \beta\right) \geq 1$ such that

$$
\int_{B_{0}}\left(M^{\sharp} u(x)\right)^{p-\varepsilon} d \mu(x) \leq C \int_{B_{0}}\left(M_{\mathrm{loc}}^{\sharp} u(x)\right)^{p-\varepsilon} d \mu(x) .
$$

Proof. Recall that

$$
\int_{B_{0}}\left(M^{\sharp}(x)\right)^{p-\varepsilon} d \mu(x)=(p-\varepsilon) \int_{0}^{\infty} \lambda^{p-\varepsilon} \mu\left(\left\{x \in B_{0}: M^{\sharp} u(x)>\lambda\right\}\right) \frac{d \lambda}{\lambda} .
$$

By using also the corresponding identity for the maximal function $M_{\mathrm{loc}}^{\sharp} u$, we see that it suffices to prove that inequality

$$
\mu\left(U^{\lambda}\right) \leq C_{1} \mu\left(V^{\lambda / C_{1}}\right)
$$

holds for some $C_{1}=C\left(c_{\mu}, p, \beta\right) \geq 1$. Indeed, then one can choose $C=C_{1}^{1+p}$. We will now show how inequality (4.11) follows from an adaptation of [10, Lemma 12.3.1]. However, the simple but tedious modification of the last rather short lemma is left to the interested reader.

Fix $x \in B_{0}$ and let us consider any ball $B=B\left(x_{B}, r_{B}\right)$ which satisfies the two conditions $x \in B$ and $256 B=B\left(x_{B}, 256 r_{B}\right) \subset B_{0}$. By the covering condition (W1) there is a Whitney ball

\footnotetext{
${ }^{1}$ Let us emphasize that it is important to use $Q^{*}$ in the definition for $\mathcal{B}_{Q}$ instead of $Q$.

${ }^{2}$ It is equally important to use $\mathbf{1}_{Q}$ instead of $\mathbf{1}_{Q^{*}}$ in the definition of $M_{\text {loc }}^{\sharp} u$; these are delicate matters and related to the latter selection of stopping balls with the aid of condition (W3).
} 
$Q=B\left(x_{Q}, r_{Q}\right) \in \mathcal{W}_{0}$ such that $x \in Q$. We claim that $B \subset Q^{*}$. In order to show this, we fix $y \in B \subset B\left(x, 2 r_{B}\right)$. Since $B\left(x, 255 r_{B}\right) \subset B_{0}$, we find that

$$
\begin{aligned}
d\left(y, x_{Q}\right) & \leq d(y, x)+d\left(x, x_{Q}\right)<2 r_{B}+r_{Q} \\
& \leq \frac{2}{255} \cdot \operatorname{dist}\left(x, X \backslash B_{0}\right)+\frac{\operatorname{dist}\left(x_{Q}, X \backslash B_{0}\right)}{128} \\
& \leq d\left(x, x_{Q}\right)+\frac{2}{255} \cdot \operatorname{dist}\left(x_{Q}, X \backslash B_{0}\right)+\frac{\operatorname{dist}\left(x_{Q}, X \backslash B_{0}\right)}{128} \\
& \leq \frac{\operatorname{dist}\left(x_{Q}, X \backslash B_{0}\right)}{128}+\frac{2}{255} \cdot \operatorname{dist}\left(x_{Q}, X \backslash B_{0}\right)+\frac{\operatorname{dist}\left(x_{Q}, X \backslash B_{0}\right)}{128} \\
& <\frac{\operatorname{dist}\left(x_{Q}, X \backslash B_{0}\right)}{32}=4 r_{Q} .
\end{aligned}
$$

It follows that $y \in 4 Q=Q^{*}$. We have shown that $B \subset Q^{*}$, and therefore $x \in B \in \mathcal{B}_{Q}$. Thus,

$$
M_{\mathrm{loc}}^{\sharp} u(x) \geq \mathbf{1}_{Q}(x) M_{Q}^{\sharp} u(x) \geq\left(\frac{1}{\operatorname{diam}(B)^{\beta p}} f_{B}\left|u(y)-u_{B}\right|^{p} d \mu(y)\right)^{1 / p} .
$$

With the aid of this estimate, the distributional inequality (4.11) follows from an adaptation of [10, Lemma 12.3.1] that, in turn, is based upon [12, Lemma 3.2.1].

The following lemma is a slight variant of [7, Lemma 3.6].

Lemma 4.12. Fix $\lambda>0$ and $Q \in \mathcal{W}_{0}$. Then inequality

$$
|u(x)-u(y)| \leq C\left(\beta, c_{\mu}\right) \lambda d(x, y)^{\beta}
$$

holds whenever $x, y \in Q^{*} \backslash U^{\lambda}$.

Proof. Let us remark that the property (W6) is used below without further notice. Fix $\lambda>0$, $Q \in \mathcal{W}_{0}$ and $x, y \in Q^{*} \backslash U^{\lambda}$. Write $d=d(x, y)$. Since $Q^{*} \subset B_{0}$, it suffices to prove that

$$
|u(x)-u(y)| \leq C\left(\beta, c_{\mu}\right) d(x, y)^{\beta}\left(M^{\sharp} u(x)+M^{\sharp} u(y)\right) .
$$

We first consider a point $z \in Q^{*}$ and a radius $0<r \leq 2 \operatorname{diam}\left(Q^{*}\right)$. Write $B_{i}=B\left(z, 2^{-i} r\right) \in \mathcal{B}_{0}$ for each $i \in\{0,1, \ldots\}$. Then, with the standard 'telescoping' argument, see for instance the proof of [7, Lemma 3.6], we obtain

$$
\begin{aligned}
\left|u(z)-u_{B(z, r)}\right| & \leq c_{\mu} \sum_{i=0}^{\infty} f_{B_{i}}\left|u-u_{B_{i}}\right| d \mu \\
& \leq c_{\mu} \sum_{i=0}^{\infty} 2^{\beta(1-i)} r^{\beta}\left(\frac{1}{\operatorname{diam}\left(B_{i}\right)^{\beta p}} f_{B_{i}}\left|u-u_{B_{i}}\right|^{p} d \mu\right)^{1 / p} \\
& \leq c_{\mu} M^{\sharp} u(z) \cdot \sum_{i=0}^{\infty} 2^{\beta(1-i)} r^{\beta} \leq C\left(\beta, c_{\mu}\right) r^{\beta} M^{\sharp} u(z) .
\end{aligned}
$$

As a consequence, since $y \in Q^{*}$ and $0<d=d(x, y) \leq \operatorname{diam}\left(Q^{*}\right)$,

$$
\begin{aligned}
\left|u(y)-u_{B(x, d)}\right| & \leq\left|u(y)-u_{B(y, 2 d)}\right|+\left|u_{B(y, 2 d)}-u_{B(x, d)}\right| \\
& \leq C\left(\beta, c_{\mu}\right) d^{\beta} M^{\sharp} u(y)+\frac{\mu(B(y, 2 d))}{\mu(B(x, d))} f_{B(y, 2 d)}\left|u-u_{B(y, 2 d)}\right| d \mu \\
& \leq C\left(\beta, c_{\mu}\right) d^{\beta}\left[M^{\sharp} u(y)+\left(\frac{1}{\operatorname{diam}(B(y, 2 d))^{\beta p}} f_{B(y, 2 d)}\left|u-u_{B(y, 2 d)}\right|^{p} d \mu\right)^{1 / p}\right] \\
& \leq C\left(\beta, c_{\mu}\right) d^{\beta} M^{\sharp} u(y) .
\end{aligned}
$$

It follows that

$$
|u(x)-u(y)| \leq\left|u(x)-u_{B(x, d)}\right|+\left|u_{B(x, d)}-u(y)\right| \leq C\left(\beta, c_{\mu}\right) d^{\beta}\left(M^{\sharp} u(x)+M^{\sharp} u(y)\right),
$$

which is the desired inequality (4.13). 


\subsection{Stopping construction}

The following stopping construction is needed for each Whitney ball separately. Fix a Whitney ball $Q \in \mathcal{W}_{0}$. The number

$$
\lambda_{Q}=\left(\frac{1}{\operatorname{diam}\left(Q^{*}\right)^{\beta p}} f_{Q^{*}}\left|u(y)-u_{Q^{*}}\right|^{p} d \mu(y)\right)^{1 / p}
$$

serves as a certain treshold value. Fix a level $\lambda>\lambda_{Q} / 2$. We will construct a stopping family $\mathcal{S}_{\lambda}(Q)$ of balls whose 5-dilations, in particular, cover the set $Q^{\lambda}$; recall the definition from (4.9). As a first step towards the stopping balls, let $B \in \mathcal{B}_{Q}$ be such that $B \cap Q \neq \emptyset$. The parent ball of $B$ is then defined to be $\pi(B)=2 B$ if $2 B \subset Q^{*}$ and $\pi(B)=Q^{*}$ otherwise. Observe that $B \subset \pi(B) \in \mathcal{B}_{Q}$ and $\pi(B) \cap Q \neq \emptyset$ so that the grandparent $\pi(\pi(B))$ is well defined, and so on and so forth. Moreover, by inequalities (2.1) and (2.3), and property (W3) if needed, we have $\mu(\pi(B)) \leq c_{\mu}^{5} \mu(B)$ and $\operatorname{diam}(\pi(B)) \leq 16 \operatorname{diam}(B)$

Now we come to the actual stopping argument. Let us fix a point $x \in Q^{\lambda} \subset Q$. If $\lambda_{Q} / 2<$ $\lambda<\lambda_{Q}$, then we choose $B_{x}=Q^{*} \in \mathcal{B}_{Q}$. If $\lambda \geq \lambda_{Q}$, then by using the condition $x \in Q^{\lambda}$ we first choose a starting ball $B$, with $x \in B \in \mathcal{B}_{Q}$, such that

$$
\lambda<\left(\frac{1}{\operatorname{diam}(B)^{\beta p}} f_{B}\left|u(y)-u_{B}\right|^{p} d \mu(y)\right)^{1 / p} .
$$

We continue by looking at the balls $B \subset \pi(B) \subset \pi(\pi(B)) \subset \cdots$ and we stop at the first ball among them, denoted by $B_{x} \in \mathcal{B}_{Q}$, that satisfies the following stopping conditions:

$$
\left\{\begin{array}{l}
\lambda<\left(\frac{1}{\operatorname{diam}\left(B_{x}\right)^{\beta p}} f_{B_{x}}\left|u(y)-u_{B_{x}}\right|^{p} d \mu(y)\right)^{1 / p} \\
\left(\frac{1}{\operatorname{diam}\left(\pi\left(B_{x}\right)\right)^{\beta p}} f_{\pi\left(B_{x}\right)}\left|u(y)-u_{\pi\left(B_{x}\right)}\right|^{p} d \mu(y)\right)^{1 / p} \leq \lambda .
\end{array}\right.
$$

The inequality $\lambda \geq \lambda_{Q}$ in combination with assumption $B_{0} \subsetneq X$ ensures that there always is such a stopping ball. In both cases above, the chosen ball $B_{x}^{\lambda}=B_{x} \in \mathcal{B}_{Q}$ contains the point $x$ and satisfies inequalities

$$
\lambda<\left(\frac{1}{\operatorname{diam}\left(B_{x}^{\lambda}\right)^{\beta p}} f_{B_{x}^{\lambda}}\left|u(y)-u_{B_{x}^{\lambda}}\right|^{p} d \mu(y)\right)^{1 / p} \leq 32 c_{\mu}^{5 / p} \lambda .
$$

Now, by using the $5 r$-covering lemma, we obtain a countable disjoint family

$$
\mathcal{S}_{\lambda}(Q) \subset\left\{B_{x}^{\lambda}: x \in Q^{\lambda}\right\}, \quad \lambda>\lambda_{Q} / 2,
$$

of stopping balls such that $Q^{\lambda} \subset \cup_{B \in \mathcal{S}_{\lambda}(Q)} 5 B$. Let us remark that, by the condition (W4) and stopping inequality (4.14), we have $B \subset U_{Q^{*}}^{\lambda}=U^{\lambda} \cap Q^{*}$ if $B \in \mathcal{S}_{\lambda}(Q)$ and $\lambda>\lambda_{Q} / 2$.

\subsection{Auxiliary local results}

We prove two technical results: Lemma 4.15 and Lemma 4.23. Even though the following lemma is a counterpart of [12, Lemma 3.1.2], the adaptation to our setting is non-trivial. Recall that $k \in \mathbb{N}$ is a fixed number and $\alpha=\beta p^{2} /(2(s+\beta p))>0$ with $s=\log _{2} c_{\mu}>0$.

Lemma 4.15. Suppose that $Q \in \mathcal{W}_{0}$ and let $\lambda>\lambda_{Q} / 2$. Then inequality

$$
\begin{aligned}
& \frac{1}{\operatorname{diam}(B)^{\beta p}} \int_{U_{B}^{2^{k} \lambda}}\left|u(x)-u_{B \backslash U^{2^{k} \lambda}}\right|^{p} d \mu(x) \\
& \leq C\left(p, c_{\mu}\right) 2^{-k \alpha}\left(2^{k} \lambda\right)^{p} \mu\left(U_{B}^{2^{k} \lambda}\right)+\frac{C\left(p, c_{\mu}\right)}{\operatorname{diam}(B)^{\beta p}} \int_{B \backslash U^{2^{k} \lambda}}\left|u(x)-u_{B \backslash U^{2^{k} \lambda}}\right|^{p} d \mu(x)
\end{aligned}
$$

holds whenever $B \in \mathcal{S}_{\lambda}(Q)$ is such that $\mu\left(U_{B}^{2^{k} \lambda}\right)<\mu(B) / 2$. 
Proof. Fix $\lambda>\lambda_{Q} / 2$ and let $B \in \mathcal{S}_{\lambda}(Q)$ be such that $\mu\left(U_{B}^{2^{k} \lambda}\right)<\mu(B) / 2$. Fix $x \in U_{B}^{2^{k} \lambda} \subset B$. Consider the function $h:(0, \infty) \rightarrow \mathbb{R}$,

$$
r \mapsto h(r)=\frac{\mu\left(U_{B}^{2^{k} \lambda} \cap B(x, r)\right)}{\mu(B \cap B(x, r))}=\frac{\mu\left(U_{B}^{2^{k} \lambda} \cap B(x, r)\right)}{\mu(B(x, r))} \cdot\left(\frac{\mu(B \cap B(x, r))}{\mu(B(x, r))}\right)^{-1} .
$$

By Lemma 2.4 and the fact that $B$ is open, we find that $h:(0, \infty) \rightarrow \mathbb{R}$ is continuous. Since $h(r)=1$ for small values of $r>0$, and $h(r)<1 / 2$ for $r>\operatorname{diam}(B)$, we find that $h\left(r_{x}\right)=1 / 2$ for some $0<r_{x} \leq \operatorname{diam}(B)$. We write $B_{x}^{\prime}=B\left(x, r_{x}\right)$. Then

$$
\frac{\mu\left(U_{B}^{2^{k} \lambda} \cap B_{x}^{\prime}\right)}{\mu\left(B \cap B_{x}^{\prime}\right)}=h\left(r_{x}\right)=\frac{1}{2}
$$

and

$$
\frac{\mu\left(\left(B \backslash U^{2^{k} \lambda}\right) \cap B_{x}^{\prime}\right)}{\mu\left(B \cap B_{x}^{\prime}\right)}=1-\frac{\mu\left(U_{B}^{2^{k} \lambda} \cap B_{x}^{\prime}\right)}{\mu\left(B \cap B_{x}^{\prime}\right)}=1-h\left(r_{x}\right)=\frac{1}{2} .
$$

Let $\mathcal{G}_{\lambda}$ be a countable disjoint subfamily of $\left\{B_{x}^{\prime}: x \in U_{B}^{2^{k} \lambda}\right\}$ such that $U_{B}^{2^{k} \lambda} \subset \cup_{B^{\prime} \in \mathcal{G}_{\lambda}} 5 B^{\prime}$. Then (4.17) and (4.18) hold for every ball $B^{\prime} \in \mathcal{G}_{\lambda}$; indeed, by denoting $B_{I}^{\prime}=U_{B}^{2^{k} \lambda} \cap B^{\prime}$ and $B_{O}^{\prime}=\left(B \backslash U^{2^{k} \lambda}\right) \cap B^{\prime}$, we have the following comparison identities:

$$
\mu\left(B_{I}^{\prime}\right)=\frac{\mu\left(B \cap B^{\prime}\right)}{2}=\mu\left(B_{O}^{\prime}\right),
$$

where all the measures are strictly positive. These identities are important and they are used several times throughout the remainder of this proof.

We multiply the left-hand side of (4.16) by $\operatorname{diam}(B)^{\beta p}$ and then estimate as follows:

$$
\begin{aligned}
\int_{U_{B}^{2^{k} \lambda}} \mid & u-\left.u_{B \backslash U^{2} k_{\lambda}}\right|^{p} d \mu \leq \sum_{B^{\prime} \in \mathcal{G}_{\lambda}} \int_{5 B^{\prime} \cap B}\left|u-u_{B \backslash U^{2^{k_{\lambda}}}}\right|^{p} d \mu \\
& \leq 2^{p-1} \sum_{B^{\prime} \in \mathcal{G}_{\lambda}} \mu\left(5 B^{\prime} \cap B\right)\left|u_{B_{O}^{\prime}}-u_{B \backslash U^{2} k_{\lambda}}\right|^{p}+2^{p-1} \sum_{B^{\prime} \in \mathcal{G}_{\lambda}} \int_{5 B^{\prime} \cap B}\left|u-u_{B_{O}^{\prime}}\right|^{p} d \mu .
\end{aligned}
$$

By (2.1) and Lemma 2.5, we find that $\mu\left(5 B^{\prime} \cap B\right) \leq \mu\left(8 B^{\prime}\right) \leq c_{\mu}^{6} \mu\left(B \cap B^{\prime}\right)$ if $B^{\prime} \in \mathcal{G}_{\lambda}$. Hence, by the comparison identities (4.19),

$$
\begin{gathered}
2^{p-1} \sum_{B^{\prime} \in \mathcal{G}_{\lambda}} \mu\left(5 B^{\prime} \cap B\right)\left|u_{B_{O}^{\prime}}-u_{B \backslash U^{2^{k_{\lambda}}}}\right|^{p} \leq C\left(p, c_{\mu}\right) \sum_{B^{\prime} \in \mathcal{G}_{\lambda}} \mu\left(B_{O}^{\prime}\right) f_{B_{O}^{\prime}}\left|u-u_{B \backslash U^{2} k_{\lambda}}\right|^{p} d \mu \\
\quad=C\left(p, c_{\mu}\right) \sum_{B^{\prime} \in \mathcal{G}_{\lambda}} \int_{B_{O}^{\prime}}\left|u-u_{B \backslash U^{2^{k_{\lambda}}}}\right|^{p} d \mu \leq C\left(p, c_{\mu}\right) \int_{B \backslash U^{2^{k} \lambda}}\left|u-u_{B \backslash U^{2^{k}}{ }^{\prime}}\right|^{p} d \mu .
\end{gathered}
$$

This concludes our analysis of the 'easy term' in (4.20). In order to treat the remaining term therein, we do need some preparations.

Let us fix a ball $B^{\prime} \in \mathcal{G}_{\lambda}$ that satisfies $\int_{5 B^{\prime} \cap B}\left|u-u_{B_{O}^{\prime}}\right|^{p} d \mu \neq 0$. We claim that

$$
f_{5 B^{\prime} \cap B}\left|u-u_{B_{O}^{\prime}}\right|^{p} d \mu \leq C\left(p, c_{\mu}\right) 2^{-k \alpha}\left(2^{k} \lambda\right)^{p} \operatorname{diam}(B)^{\beta p} .
$$

In order to prove this inequality, we fix a number $m \in \mathbb{R}$ such that

$$
\left(2^{m} \lambda\right)^{p} \operatorname{diam}\left(5 B^{\prime}\right)^{\beta p}=f_{5 B^{\prime} \cap B}\left|u-u_{B_{O}^{\prime}}\right|^{p} d \mu .
$$

Let us first consider the case $m<k / 2$. Then $m-k<-k / 2$, and since always $\alpha<p / 2$, the desired inequality (4.22) is obtained in this case as follows:

$$
\begin{aligned}
f_{5 B^{\prime} \cap B}\left|u-u_{B_{O}^{\prime}}\right|^{p} d \mu & =2^{(m-k) p}\left(2^{k} \lambda\right)^{p} \operatorname{diam}\left(5 B^{\prime}\right)^{\beta p} \\
& \leq 10^{p} 2^{-k p / 2}\left(2^{k} \lambda\right)^{p} \operatorname{diam}(B)^{\beta p} \leq C(p) 2^{-k \alpha}\left(2^{k} \lambda\right)^{p} \operatorname{diam}(B)^{\beta p} .
\end{aligned}
$$


Next we consider the case $k / 2 \leq m$. By comparison identities (4.19) and Lemma 2.5,

$$
\begin{aligned}
f_{5 B^{\prime} \cap B}\left|u-u_{B_{O}^{\prime}}\right|^{p} d \mu & \leq 2^{p-1} f_{5 B^{\prime} \cap B}\left|u-u_{5 B^{\prime}}\right|^{p} d \mu+2^{p-1}\left|u_{5 B^{\prime}}-u_{B_{O}^{\prime}}\right|^{p} \\
& \leq 2^{p+1} c_{\mu}^{6} f_{5 B^{\prime}}\left|u-u_{5 B^{\prime}}\right|^{p} d \mu \leq 2^{p+1} c_{\mu}^{6}\left(2^{k} \lambda\right)^{p} \operatorname{diam}\left(5 B^{\prime}\right)^{\beta p}
\end{aligned}
$$

where the last step follows from condition (W5) and the fact that $5 B^{\prime} \supset B_{O}^{\prime} \neq \emptyset$. It follows that $2^{m p} \leq 2^{p+1} c_{\mu}^{6} 2^{k p}$. On the other hand, we have

$$
\begin{aligned}
\left(2^{m} \lambda\right)^{p} \operatorname{diam}\left(5 B^{\prime}\right)^{\beta p} \mu\left(B^{\prime} \cap B\right) & \leq \int_{5 B^{\prime} \cap B}\left|u-u_{B_{O}^{\prime}}\right|^{p} d \mu \\
& \leq 2^{p-1} \int_{5 B^{\prime} \cap B}\left|u-u_{B}\right|^{p} d \mu+2^{p-1} \mu\left(5 B^{\prime} \cap B\right)\left|u_{B_{O}^{\prime}}-u_{B}\right|^{p} \\
& \leq 2^{p+1} c_{\mu}^{6} \int_{B}\left|u-u_{B}\right|^{p} d \mu \leq 2 \cdot 64^{p} c_{\mu}^{11} \lambda^{p} \operatorname{diam}(B)^{\beta p} \mu(B),
\end{aligned}
$$

where the last step follows from the fact that $B \in \mathcal{S}_{\lambda}(Q)$ in combination with inequality (4.14). In particular, if $s=\log _{2} c_{\mu}$ then by inequality (2.2) and Lemma 2.5, we obtain that

$$
\begin{aligned}
\left(\frac{\operatorname{diam}\left(5 B^{\prime}\right)}{\operatorname{diam}(B)}\right)^{s+\beta p} & \leq 20^{s} \frac{\operatorname{diam}\left(5 B^{\prime}\right)^{\beta p} \mu\left(B^{\prime}\right)}{\operatorname{diam}(B)^{\beta p} \mu(B)} \leq 20^{s} c_{\mu}^{3} \frac{\operatorname{diam}\left(5 B^{\prime}\right)^{\beta p} \mu\left(B^{\prime} \cap B\right)}{\operatorname{diam}(B)^{\beta p} \mu(B)} \\
& \leq 2 \cdot 64^{p} 20^{s} c_{\mu}^{14} 2^{-m p} \leq 2 \cdot 64^{p} 20^{s} c_{\mu}^{14} 2^{-k p / 2} .
\end{aligned}
$$

This, in turn, implies that

$$
\left(\frac{\operatorname{diam}\left(5 B^{\prime}\right)}{\operatorname{diam}(B)}\right)^{\beta p} \leq 2 \cdot 64^{p} 20^{s} c_{\mu}^{14} 2^{\frac{-k \beta p^{2}}{2(s+\beta p)}}=C\left(p, c_{\mu}\right) 2^{-k \alpha}
$$

Combining the above estimates, we see that

$$
f_{5 B^{\prime} \cap B}\left|u-u_{B_{O}^{\prime}}\right|^{p} d \mu=\left(2^{m} \lambda\right)^{p} \operatorname{diam}\left(5 B^{\prime}\right)^{\beta p} \leq C\left(p, c_{\mu}\right) 2^{-k \alpha}\left(2^{k} \lambda\right)^{p} \operatorname{diam}(B)^{\beta p} .
$$

That is, inequality (4.22) holds also in the present case $k / 2 \leq m$.

By using Lemma 2.5 and inequalities (4.19) and (4.22), we can now estimate the second term in (4.20) as follows:

$$
\begin{aligned}
2^{p-1} \sum_{B^{\prime} \in \mathcal{G}_{\lambda}} \int_{5 B^{\prime} \cap B}\left|u-u_{B_{O}^{\prime}}\right|^{p} d \mu & \leq 2^{p} c_{\mu}^{6} \sum_{B^{\prime} \in \mathcal{G}_{\lambda}} \mu\left(B_{I}^{\prime}\right) f_{5 B^{\prime} \cap B}\left|u-u_{B_{O}^{\prime}}\right|^{p} d \mu \\
& \leq C\left(p, c_{\mu}\right) 2^{-k \alpha}\left(2^{k} \lambda\right)^{p} \operatorname{diam}(B)^{\beta p} \sum_{B^{\prime} \in \mathcal{G}_{\lambda}} \mu\left(B_{I}^{\prime}\right) \\
& \leq C\left(p, c_{\mu}\right) 2^{-k \alpha}\left(2^{k} \lambda\right)^{p} \operatorname{diam}(B)^{\beta p} \mu\left(U_{B}^{2^{k} \lambda}\right) .
\end{aligned}
$$

Inequality (4.16) follows by collecting the above estimates.

The following lemma is essential for the proof of Theorem 4.3, and it is the only place in the proof where the $(p, p)$-Poincaré inequality is needed - and, moreover, this inequality is applied only a single time.

Lemma 4.23. Fix a Whitney ball $Q \in \mathcal{W}_{0}$. Then inequality

$$
\lambda^{p} \mu\left(Q^{\lambda}\right) \leq C\left(\beta, p, c_{\mu}\right)\left[\frac{\left(\lambda 2^{k}\right)^{p}}{2^{k \alpha}} \mu\left(U_{Q^{*}}^{2^{k} \lambda}\right)+\frac{K_{p, p}}{k^{p}} \sum_{j=k}^{2 k-1}\left(\lambda 2^{j}\right)^{p} \mu\left(U_{Q^{*}}^{2^{j} \lambda}\right)+K_{p, p} \int_{U_{Q^{*}}^{\lambda} \backslash U^{4^{k} \lambda}} g^{p} d \mu\right]
$$

holds for each $\lambda>\lambda_{Q} / 2$ and every $g \in \mathcal{D}(u)$. 
Proof. Fix $\lambda>\lambda_{Q} / 2$ and $g \in \mathcal{D}(u)$. By the doubling condition (2.1),

$$
\lambda^{p} \mu\left(Q^{\lambda}\right) \leq \lambda^{p} \sum_{B \in \mathcal{S}_{\lambda}(Q)} \mu(5 B) \leq c_{\mu}^{3} \sum_{B \in \mathcal{S}_{\lambda}(Q)} \lambda^{p} \mu(B) .
$$

Recall also that $B \subset U_{Q^{*}}^{\lambda}=U^{\lambda} \cap Q^{*}$ if $B \in \mathcal{S}_{\lambda}(Q)$. Therefore, and using the fact that $\mathcal{S}_{\lambda}(Q)$ is a disjoint family, it suffices to prove that inequality

$$
\lambda^{p} \mu(B) \leq C\left(\beta, p, c_{\mu}\right)\left[\frac{\left(\lambda 2^{k}\right)^{p}}{2^{k \alpha}} \mu\left(U_{B}^{2^{k} \lambda}\right)+\frac{K_{p, p}}{k^{p}} \sum_{j=k}^{2 k-1}\left(\lambda 2^{j}\right)^{p} \mu\left(U_{B}^{2^{j} \lambda}\right)+K_{p, p} \int_{B \backslash U^{4^{k} \lambda}} g^{p} d \mu\right]
$$

holds for every $B \in \mathcal{S}_{\lambda}(Q)$. To this end, let us fix a ball $B \in \mathcal{S}_{\lambda}(Q)$.

If $\mu\left(U_{B}^{2^{k} \lambda}\right) \geq \mu(B) / 2$, then

$$
\lambda^{p} \mu(B) \leq 2 \lambda^{p} \mu\left(U_{B}^{2^{k} \lambda}\right)=2 \frac{\left(\lambda 2^{k}\right)^{p}}{2^{k p}} \mu\left(U_{B}^{2^{k} \lambda}\right) \leq 2 \frac{\left(\lambda 2^{k}\right)^{p}}{2^{k \alpha}} \mu\left(U_{B}^{2^{k} \lambda}\right),
$$

which suffices for the required local estimate (4.25). Let us then consider the more difficult case $\mu\left(U_{B}^{2^{k} \lambda}\right)<\mu(B) / 2$. In this case, by the stopping inequality (4.14),

$$
\begin{aligned}
\lambda^{p} \mu(B) & \leq \frac{1}{\operatorname{diam}(B)^{\beta p}} \int_{B}\left|u(x)-u_{B}\right|^{p} d \mu(x) \\
& \leq \frac{2^{p}}{\operatorname{diam}(B)^{\beta p}} \int_{X}\left(\mathbf{1}_{B \backslash U^{2^{k}}}(x)+\mathbf{1}_{U_{B}^{2^{k} \lambda}}(x)\right)\left|u(x)-u_{B \backslash U^{2^{k}}}\right|^{p} d \mu(x) .
\end{aligned}
$$

By Lemma 4.15 it suffices to estimate the integral over the set $B \backslash U^{2^{k} \lambda}=B \backslash U_{B}^{2^{k} \lambda}$; observe that the measure of this set is strictly positive. We remark that the Poincaré inequality condition (D1') will be used to estimate this integral.

Fix a number $i \in \mathbb{N}$. Recall that $B \subset Q^{*}$. Hence, it follows from Lemma 4.12 that the restriction $\left.u\right|_{B \backslash U^{2^{i} \lambda}}: B \backslash U^{2^{i} \lambda} \rightarrow \mathbb{R}$ is a $\beta$-Hölder function with a constant $\kappa_{i}=C\left(\beta, c_{\mu}\right) 2^{i} \lambda$. We can now use the McShane extension (2.6) and extend $\left.u\right|_{B \backslash U^{2^{i} \lambda}}$ to a function $u_{2^{i} \lambda}: X \rightarrow \mathbb{R}$ that is $\beta$-Hölder with the constant $\kappa_{i}$ and satisfies the restriction identity $\left.u_{2^{i} \lambda}\right|_{B \backslash U^{2^{i} \lambda}}=\left.u\right|_{B \backslash U^{2^{i} \lambda}}$.

The crucial idea that was also used by Keith-Zhong in [12] is to consider the function

$$
h(x)=\frac{1}{k} \sum_{i=k}^{2 k-1} u_{2^{i} \lambda}(x), \quad x \in X .
$$

By conditions (D2)-(D4) of the fixed $\mathcal{D}$-structure, we obtain that

$$
g_{h}=\frac{1}{k} \sum_{i=k}^{2 k-1}\left(\kappa_{i} \mathbf{1}_{U^{2^{i} \lambda} \cup B^{c}}+g \mathbf{1}_{B \backslash U^{2^{i} \lambda}}\right) \in \mathcal{D}(h) .
$$

Observe that $U_{B}^{2^{k} \lambda} \supset U_{B}^{2^{(k+1)} \lambda} \supset \cdots \supset U_{B}^{2^{(2 k-1)} \lambda} \supset U_{B}^{4^{k} \lambda}$. By using these inclusions it is straightforward to show that the following pointwise estimates are valid in $X$,

$$
\begin{aligned}
\mathbf{1}_{B} g_{h}^{p} & \leq\left(\frac{1}{k} \sum_{i=k}^{2 k-1}\left(\kappa_{i} \mathbf{1}_{U_{B}^{2^{i} \lambda}}+g \mathbf{1}_{B \backslash U^{2^{2} \lambda}}\right)\right)^{p} \\
& \leq 2^{p}\left(\frac{1}{k} \sum_{i=k}^{2 k-1} \kappa_{i} \mathbf{1}_{U_{B}^{2^{i} \lambda}}\right)^{p}+2^{p} g^{p} \mathbf{1}_{B \backslash U^{4} k_{\lambda}} \\
& \leq \frac{C\left(\beta, p, c_{\mu}\right)}{k^{p}} \sum_{j=k}^{2 k-1}\left(\sum_{i=k}^{j} 2^{i} \lambda\right)^{p} \mathbf{1}_{U_{B}^{2 j_{\lambda}}}+2^{p} g^{p} \mathbf{1}_{B \backslash U^{4} k_{\lambda}} \\
& \leq \frac{C\left(\beta, p, c_{\mu}\right)}{k^{p}} \sum_{j=k}^{2 k-1}\left(\lambda 2^{j}\right)^{p} \mathbf{1}_{U_{B}^{2 j_{\lambda}}}+2^{p} g^{p} \mathbf{1}_{B \backslash U^{4} k_{\lambda}} .
\end{aligned}
$$


Observe that $h$ coincides with $u$ on $B \backslash U^{2^{k} \lambda}$ and recall that $g_{h} \in \mathcal{D}(h)$. Hence the Poincaré inequality from condition (D1') in Theorem 3.3 implies that

$$
\begin{aligned}
& \frac{1}{\operatorname{diam}(B)^{\beta p}} \int_{B \backslash U^{2^{k} \lambda}}\left|u(x)-u_{B \backslash U^{2^{k} \lambda}}\right|^{p} d \mu(x) \leq \frac{2^{p}}{\operatorname{diam}(B)^{\beta p}} \int_{B}\left|h(x)-h_{B}\right|^{p} d \mu(x) \\
& \leq 2^{p} K_{p, p} \int_{B} g_{h}(x)^{p} d \mu(x) \\
& \leq \frac{C\left(\beta, p, c_{\mu}\right) K_{p, p}}{k^{p}} \sum_{j=k}^{2 k-1}\left(\lambda 2^{j}\right)^{p} \mu\left(U_{B}^{2^{j} \lambda}\right)+4^{p} K_{p, p} \int_{B \backslash U^{4^{k} \lambda}} g(x)^{p} d \mu(x) .
\end{aligned}
$$

The desired local inequality (4.25) follows by combining the estimates above.

\subsection{Completing proof of Theorem 4.3}

Recall that $u: X \rightarrow \mathbb{R}$ is a $\beta$-Hölder function and that $M^{\sharp} u=M_{\beta, \mathcal{B}_{0}}^{\sharp, p}$. Let us fix a function $g \in \mathcal{D}(u)$. Observe that the left-hand side of inequality (4.4) is finite. Without loss of generality, we may further assume that it is nonzero. By Lemma 4.10,

$$
\int_{B_{0}}\left(M^{\sharp} u(x)\right)^{p-\varepsilon} d \mu(x) \leq C\left(c_{\mu}, p, \beta\right) \int_{B_{0}}\left(M_{\mathrm{loc}}^{\sharp} u(x)\right)^{p-\varepsilon} d \mu(x) .
$$

Observe that

$$
\left(M_{\mathrm{loc}}^{\sharp} u(x)\right)^{p-\varepsilon} \leq \sum_{Q \in \mathcal{W}_{0}} \mathbf{1}_{Q}(x)\left(M_{Q}^{\sharp} u(x)\right)^{p-\varepsilon}
$$

for every $x \in B_{0}$. Hence,

$$
\int_{B_{0}}\left(M_{\mathrm{loc}}^{\sharp} u(x)\right)^{p-\varepsilon} d \mu(x) \leq \sum_{Q \in \mathcal{W}_{0}} \int_{Q}\left(M_{Q}^{\sharp} u(x)\right)^{p-\varepsilon} d \mu(x) .
$$

At this stage, we fix a ball $Q \in \mathcal{W}_{0}$ and write the corresponding integral as follows:

$$
\int_{Q}\left(M_{Q}^{\sharp} u(x)\right)^{p-\varepsilon} d \mu(x)=(p-\varepsilon) \int_{0}^{\infty} \lambda^{p-\varepsilon} \mu\left(Q^{\lambda}\right) \frac{d \lambda}{\lambda} .
$$

Since $Q^{\lambda}=Q=Q^{2 \lambda}$ for every $\lambda \in\left(0, \lambda_{Q} / 2\right)$, we find that

$$
\begin{aligned}
(p-\varepsilon) \int_{0}^{\lambda_{Q} / 2} \lambda^{p-\varepsilon} \mu\left(Q^{\lambda}\right) \frac{d \lambda}{\lambda} & =\frac{(p-\varepsilon)}{2^{p-\varepsilon}} \int_{0}^{\lambda_{Q} / 2}(2 \lambda)^{p-\varepsilon} \mu\left(Q^{2 \lambda}\right) \frac{d \lambda}{\lambda} \\
& \leq \frac{(p-\varepsilon)}{2^{p-\varepsilon}} \int_{0}^{\infty} \sigma^{p-\varepsilon} \mu\left(Q^{\sigma}\right) \frac{d \sigma}{\sigma} \\
& =\frac{1}{2^{p-\varepsilon}} \int_{Q}\left(M_{Q}^{\sharp} u(x)\right)^{p-\varepsilon} d \mu(x) .
\end{aligned}
$$

On the other hand, by Lemma 4.23, for each $\lambda>\lambda_{Q} / 2$,

$$
\lambda^{p-\varepsilon} \mu\left(Q^{\lambda}\right) \leq C\left(\beta, p, c_{\mu}\right) \lambda^{-\varepsilon}\left[\frac{\left(\lambda 2^{k}\right)^{p}}{2^{k \alpha}} \mu\left(U_{Q^{*}}^{2^{k} \lambda}\right)+\frac{K_{p, p}}{k^{p}} \sum_{j=k}^{2 k-1}\left(\lambda 2^{j}\right)^{p} \mu\left(U_{Q^{*}}^{2^{j} \lambda}\right)+K_{p, p} \int_{U_{Q^{*}}^{\lambda} \backslash U^{4^{k} \lambda}} g^{p} d \mu\right] .
$$

Since $p-\varepsilon>1$, it follows that

$$
\begin{aligned}
\int_{Q}\left(M_{Q}^{\sharp} u(x)\right)^{p-\varepsilon} d \mu(x) & \leq 2(p-\varepsilon) \int_{\lambda_{Q} / 2}^{\infty} \lambda^{p-\varepsilon} \mu\left(Q^{\lambda}\right) \frac{d \lambda}{\lambda} \\
& \leq C\left(\beta, p, c_{\mu}\right)\left(I_{1}(Q)+I_{2}(Q)+I_{3}(Q)\right),
\end{aligned}
$$


where

$$
\begin{aligned}
& I_{1}(Q)=\frac{2^{k \varepsilon}}{2^{k \alpha}} \int_{0}^{\infty}\left(\lambda 2^{k}\right)^{p-\varepsilon} \mu\left(U_{Q^{*}}^{2^{k} \lambda}\right) \frac{d \lambda}{\lambda} \\
& I_{2}(Q)=\frac{K_{p, p}}{k^{p}} \sum_{j=k}^{2 k-1} 2^{j \varepsilon} \int_{0}^{\infty}\left(2^{j} \lambda\right)^{p-\varepsilon} \mu\left(U_{Q^{*}}^{2^{j} \lambda}\right) \frac{d \lambda}{\lambda} \\
& I_{3}(Q)=K_{p, p} \int_{0}^{\infty} \lambda^{-\varepsilon} \int_{U_{Q^{*}}^{\lambda} \backslash U^{4^{k} \lambda}} g(x)^{p} d \mu(x) \frac{d \lambda}{\lambda} .
\end{aligned}
$$

By (W2) we have $\sum_{Q \in \mathcal{W}_{0}} \mathbf{1}_{Q^{*}} \leq C\left(c_{\mu}\right) \mathbf{1}_{B_{0}}$. Hence, we can now continue to estimate as follows. First,

$$
\begin{aligned}
\sum_{Q \in \mathcal{W}_{0}} I_{1}(Q) & \leq C\left(c_{\mu}\right) \frac{2^{k(\varepsilon-\alpha)}}{p-\varepsilon} \int_{B_{0}}\left(M^{\sharp} u(x)\right)^{p-\varepsilon} d \mu(x) \\
& \leq C\left(c_{\mu}\right) 2^{k(\varepsilon-\alpha)} \int_{B_{0}}\left(M^{\sharp} u(x)\right)^{p-\varepsilon} d \mu(x) .
\end{aligned}
$$

Second,

$$
\begin{aligned}
\sum_{Q \in \mathcal{W}_{0}} I_{2}(Q) & \leq C\left(c_{\mu}\right) \frac{K_{p, p}}{k^{p}} \sum_{j=k}^{2 k-1} 2^{j \varepsilon} \int_{0}^{\infty}\left(2^{j} \lambda\right)^{p-\varepsilon} \mu\left(U^{2^{j} \lambda}\right) \frac{d \lambda}{\lambda} \\
& \leq \frac{C\left(c_{\mu}\right) K_{p, p}}{k^{p}(p-\varepsilon)}\left(\sum_{j=k}^{2 k-1} 2^{j \varepsilon}\right) \int_{B_{0}}\left(M^{\sharp} u(x)\right)^{p-\varepsilon} d \mu \\
& \leq C\left(c_{\mu}\right) \frac{K_{p, p} 4^{k \varepsilon}}{k^{p-1}} \int_{B_{0}}\left(M^{\sharp} u(x)\right)^{p-\varepsilon} d \mu .
\end{aligned}
$$

Third, by Fubini's theorem,

$$
\begin{aligned}
\sum_{Q \in \mathcal{W}_{0}} I_{3}(Q) & \leq C\left(c_{\mu}\right) K_{p, p} \int_{B_{0} \backslash\left\{M^{\sharp} u=0\right\}}\left(\int_{0}^{\infty} \lambda^{-\varepsilon} \mathbf{1}_{U^{\lambda} \backslash U^{4} k_{\lambda}}(x) \frac{d \lambda}{\lambda}\right) g(x)^{p} d \mu(x) \\
& \leq C\left(c_{\mu}\right) C(k, \varepsilon) K_{p, p} \int_{B_{0} \backslash\left\{M^{\sharp} u=0\right\}} g(x)^{p}\left(M^{\sharp} u(x)\right)^{-\varepsilon} d \mu(x) .
\end{aligned}
$$

Combining the estimates above, we arrive at the desired conclusion.

\section{KeITH-ZhONG THEOREMS}

We consider the self-improvement properties of Poincaré inequalities involving $p$-weak upper gradients; in particular, we recover the so-called Keith-Zhong Theorem [12]; see Theorem 5.7. Moreover, we obtain a partial version of this result for $\mathcal{D}$-structures in Theorem 5.8.

Definition 5.1. Fix an exponent $1<p<\infty$. A measurable function $g: X \rightarrow[0, \infty]$ is a $p$-weak upper gradient (with respect to $X$ ) of a function $u: X \rightarrow \mathbb{R}$ if inequality

$$
\left|u(\gamma(0))-u\left(\gamma\left(\ell_{\gamma}\right)\right)\right| \leq \int_{\gamma} g d s
$$

holds for $p$-almost every curve $\gamma:\left[0, \ell_{\gamma}\right] \rightarrow X$; i.e., there exists a non-negative Borel function $\rho \in L^{p}(X)$ such that $\int_{\gamma} \rho d s=\infty$ whenever inequality (5.2) does not hold or is not defined.

We refer to $[1,9,10]$ for further information on $p$-weak upper gradients.

Fix an exponent $1<p<\infty$. For each function $u \in \operatorname{Lip}(X)$, we let $\mathcal{D}_{N}^{1, p}(u)$ be the family of all $p$-weak upper gradients $g \in L_{\mathrm{loc}}^{p}(X)$ of $u$; by $g \in L_{\mathrm{loc}}^{p}(X)$ we mean that for each $x \in X$ there exists $r_{x}>0$ such that $g \in L^{p}\left(B\left(x, r_{x}\right)\right)$. The properties (D2) and (D3) in Definition 3.1 are rather well known, see for instance [1, Corollary 1.39]. The property (D4) with $\beta=1$ is a 
consequence of a so-called 'Glueing lemma', we refer to [1, Lemma 2.19, Remark 2.28]. However, the $(1, p)$-Poincaré inequality condition (D1), with $\beta=1$, is not always valid, and therefore we need to assume this in some of the forthcoming results.

Beyond these properties (D1)-(D4), we also need some other observations. The above family $\mathcal{D}_{N}^{1, p}(u)$ has the following minimality property: if $u \in \operatorname{Lip}(X)$, then there exists a $p$-weak upper gradient $g_{u} \in \mathcal{D}_{N}^{1, p}(u)$ such that $g_{u} \leq g$ almost everywhere if $g \in \mathcal{D}_{N}^{1, p}(u)$; see [1, Theorem 2.25]. Moreover, this minimal p-weak upper gradient $g_{u}$ is unique up to sets of measure zero in $X$. The following result is an adaptation of [14, Lemma 4.7]; see also [11]. The proof below relies on a localization property of the minimal $p$-weak upper gradient to open sets (e.g. to balls $B_{0}$ in $X$ ). At this stage, the reader is encouraged to recall Definition (4.1).

Lemma 5.3. Let $1<p<\infty$. Let $B_{0} \subset X$ be a ball and $\mathcal{B}_{0}=\left\{B(x, r): B(x, 2 r) \subset B_{0}\right\}$. Suppose that $u: X \rightarrow \mathbb{R}$ is a Lipschitz function and let $g_{u} \in L_{\mathrm{loc}}^{p}(X)$ be its minimal p-weak upper gradient. Then inequality

$$
g_{u}(x) \leq C\left(c_{\mu}\right) M_{1, \mathcal{B}_{0}}^{\sharp, p} u(x)
$$

holds for almost every $x \in B_{0}$.

Proof. In the proof, we only consider the difficult case $B_{0} \neq X$; the case $B_{0}=X$ is similar. Let $u: X \rightarrow \mathbb{R}$ be Lipschitz, with a constant $\kappa>0$. Write $g=C\left(1, c_{\mu}\right) M_{1, \mathcal{B}_{0}}^{\sharp, p} u$, where the constant $C\left(1, c_{\mu}\right)>0$ is as in the proof of Lemma 4.12. First we show that $\left.4 g\right|_{B_{0}}$ is a $p$-weak upper gradient of $\left.u\right|_{B_{0}}$ with respect to $B_{0}$.

To begin with, we observe that $\left\{y \in B_{0}: g(y)>\lambda\right\}$ is an open set if $\lambda \in \mathbb{R}$. Hence, the function $\left.g\right|_{B_{0}}$ is Borel in $B_{0}$. Fix a curve $\gamma:\left[0, \ell_{\gamma}\right] \rightarrow B_{0} \subset X$, and then fix a natural number $n \geq 2$ satisfying condition

$$
n>2 \ell_{\gamma}\left(\frac{\operatorname{dist}\left(\gamma\left[0, \ell_{\gamma}\right], X \backslash B_{0}\right)}{128}\right)^{-1}>0 .
$$

At the end, we will let $n$ tend to infinity. We consider the covering $\left[0, \ell_{\gamma}\right]=\cup_{i=0}^{n-1}\left[t_{i}, t_{i+1}\right]$, where each $t_{j}=j \ell_{\gamma} / n$. Write $\gamma_{i}=\left.\gamma\right|_{\left[t_{i}, t_{i+1}\right]}$ and $\left|\gamma_{i}\right|=\gamma\left[t_{i}, t_{i+1}\right] \subset B_{0}$ for each $i=0, \ldots, n-1$. For each such $i$ we pick $x_{i}=x_{i}(n) \in\left|\gamma_{i}\right|$ such that

$$
g\left(x_{i}\right) \leq f_{\gamma_{i}} g d s
$$

Consider a fixed $i=0, \ldots, n-2$. Note first that

$$
d\left(x_{i}, x_{i+1}\right) \leq \ell\left(\gamma_{i}\right)+\ell\left(\gamma_{i+1}\right)=2 \ell_{\gamma} / n=2 \ell\left(\gamma_{i}\right)=2 \ell\left(\gamma_{i+1}\right) .
$$

Moreover, since $B_{0} \subsetneq X$, we can choose a Whitney ball $Q_{i} \in \mathcal{W}\left(B_{0}\right)$ such that $x_{i} \in Q_{i}$; we refer to $\S 4.2$. By using inequalities (5.5) and (5.6), it is straightforward to show that $x_{i}, x_{i+1} \in Q_{i}^{*}$. Hence, proceeding as in the proof of Lemma 4.12, we see that

$$
\left|u\left(x_{i}\right)-u\left(x_{i+1}\right)\right| \leq d\left(x_{i}, x_{i+1}\right)\left(g\left(x_{i}\right)+g\left(x_{i+1}\right)\right) .
$$

Thus, we obtain that

$$
\begin{aligned}
\left|u\left(x_{0}\right)-u\left(x_{n-1}\right)\right| & \leq \sum_{i=0}^{n-2}\left|u\left(x_{i}\right)-u\left(x_{i+1}\right)\right| \\
& \leq \sum_{i=0}^{n-2} d\left(x_{i}, x_{i+1}\right)\left(g\left(x_{i}\right)+g\left(x_{i+1}\right)\right) \\
& \leq 4 \sum_{i=0}^{n-1} \ell\left(\gamma_{i}\right) f_{\gamma_{i}} g d s=4 \int_{\gamma} g d s .
\end{aligned}
$$


By taking $n \rightarrow \infty$ and using the continuity of $u$, together with the facts that $x_{0}(n) \rightarrow \gamma(0)$ and $x_{n-1}(n) \rightarrow \gamma\left(\ell_{\gamma}\right)$, we conclude that

$$
\left|u(\gamma(0))-u\left(\gamma\left(\ell_{\gamma}\right)\right)\right| \leq \int_{\gamma} 4 g d s .
$$

This inequality shows that $\left.4 g\right|_{B_{0}}$ is a $p$-weak upper gradient of $\left.u\right|_{B_{0}}$ with respect to $B_{0}$, and since $0 \leq 4 g \leq 4 C\left(1, c_{\mu}\right) \kappa$, it also holds that $\left.4 g\right|_{B_{0}} \in L^{p}\left(B_{0}\right)$. Inequality (5.4) for $C\left(c_{\mu}\right)=4 C\left(1, c_{\mu}\right)$ now follows from the fact that the restriction $\left.g_{u}\right|_{B_{0}}$ is the minimal $p$-weak upper gradient of $\left.u\right|_{B_{0}}$ with respect to the (open) ball $B_{0}$; we refer to [1, Lemma 2.23].

The following result is now a consequence of our main result, Theorem 4.3. This result can be further strenghtened by using Theorem 3.6, but we leave details to the interested reader.

Theorem 5.7. Suppose that $X$ is a geodesic space and fix $1<p<\infty$. Suppose that there are constants $K>0$ and $\tau \geq 1$ such that the $(1, p)$-Poincaré inequality

$$
f_{B}\left|u(x)-u_{B}\right| d \mu(x) \leq K^{1 / p} \operatorname{diam}(B)\left(f_{\tau B} g(x)^{p} d \mu(x)\right)^{1 / p}
$$

holds whenever $B$ is a ball in $X$ and $g \in L_{\mathrm{loc}}^{p}(X)$ is a p-weak upper gradient of $u \in \operatorname{Lip}(X)$. Then there exists a number $0<\varepsilon<p-1$ and a constant $C>0$, both of which are quantitative, such that inequality

$$
\left(f_{B}\left|u(x)-u_{B}\right|^{p} d \mu(x)\right)^{1 / p} \leq C \operatorname{diam}(B)\left(f_{2 B} g(x)^{p-\varepsilon} d \mu(x)\right)^{1 /(p-\varepsilon)}
$$

holds whenever $B \subset X$ is a ball, $u \in \operatorname{Lip}(X)$ and $g \in L_{\mathrm{loc}}^{p}(X)$ is a p-weak upper gradient of $u$.

Proof. From the above considerations and the standing assumptions, it follows that the family $\left\{\mathcal{D}_{N}^{1, p}(u): u \in \operatorname{Lip}(X)\right\}$ is a $\mathcal{D}$-structure in $X$, with exponents $p$ and $\beta=1$, and constants $K>0$ and $\tau \geq 1$. This allows us to fix $0<\varepsilon<\varepsilon_{0}$ as in Corollary 4.5. Fix also a ball $B \subset X$, and write $B_{0}=2 B$ and $\mathcal{B}_{0}=\left\{B(x, r): B(x, 2 r) \subset B_{0}\right\}$. Since $B \in \mathcal{B}_{0}$, we have

$$
\mu(B)\left(\frac{1}{\operatorname{diam}(B)^{p}} f_{B}\left|u(x)-u_{B}\right|^{p} d \mu(x)\right)^{(p-\varepsilon) / p} \leq \int_{B_{0}}\left(M_{1, \mathcal{B}_{0}}^{\sharp, p} u\right)^{p-\varepsilon} d \mu .
$$

We apply Corollary 4.5 with the ball $B_{0} \subset X$ and with the minimal $p$-weak upper gradient $g_{u} \in L_{\mathrm{loc}}^{p}(X)$ of $u$. Lemma 5.3 is needed to obtain the estimate

$$
\int_{B_{0} \backslash\left\{M_{1, \mathcal{B}_{0}}^{\sharp, p} u=0\right\}} g_{u}^{p}\left(M_{1, \mathcal{B}_{0}}^{\sharp, p} u\right)^{-\varepsilon} d \mu \leq C\left(c_{\mu}, \varepsilon\right) \int_{B_{0}} g_{u}^{p-\varepsilon} d \mu
$$

for the right-hand side of (4.6). At the end we use the fact that $g_{u}^{p-\varepsilon} \leq g^{p-\varepsilon}$ almost everywhere if $g \in L_{\text {loc }}^{p}(X)$ is any $p$-weak upper gradient of $u$.

Along the same lines, we can also prove a version of the Keith-Zhong theorem for general $\mathcal{D}$-structures in geodesic spaces. This result is stated in Theorem 5.8 below. This result is a true generalization of Theorem 5.7 but it is unknown to the authors whether the additional minimality condition in the statement below can be removed.

Theorem 5.8. Suppose that we are given a $\mathcal{D}$-structure in a geodesic space $X$, with exponents $1<p<\infty$ and $0<\beta \leq 1$, and constants $K>0$ and $\tau \geq 1$. Fix $\eta>0$. Then there exists a number $0<\varepsilon<p-1$ and a constant $C>0$, both of which are quantitative, such that inequality

$$
\left(f_{B}\left|u(x)-u_{B}\right|^{p} d \mu(x)\right)^{1 / p} \leq C \operatorname{diam}(B)^{\beta}\left(f_{2 B} g(x)^{p-\varepsilon} d \mu(x)\right)^{1 /(p-\varepsilon)}
$$

holds whenever $B \subset X$ is a ball, $u \in \operatorname{Lip}_{\beta}(X)$ and $g \in \mathcal{D}(u)$ satisfies the following minimality condition: $g \leq \eta M_{\beta, \mathcal{B}_{0}}^{\sharp, p}$ almost everywhere in $B_{0}=2 B$.

Theorem 5.8 can also be further strenghtened by using Theorem 3.6, but again we leave details to the interested reader. 


\section{Axiomatic Sobolev spaces}

A given $\mathcal{D}$-structure gives rise to a Sobolev space; cf. [5]. Our main result in this section is a certain norm-equivalence for such spaces, Theorem 6.2.

\subsection{Sobolev spaces and $\mathcal{D}$-structures}

Let us begin with the definition of an abstract Sobolev space that is defined in terms of a $\mathcal{D}$ structure. Our treatment is inspired by [5]. See also [6, 15] for further references on this type of abstract Sobolev spaces.

Definition 6.1. Given a $\mathcal{D}$-structure $\mathcal{D}$ in $X$, with exponents $1 \leq p<\infty$ and $0<\beta \leq 1$, the associated Sobolev space $W_{\beta}^{p}(X, \mathcal{D})$ is the completion [13] of the vector space

$$
\left\{u \in \operatorname{Lip}_{\beta}(X):\|u\|_{W_{\beta}^{p}(X, \mathcal{D})}<\infty\right\}
$$

that is equipped with the norm ${ }^{3}$

$$
\|u\|_{W_{\beta}^{p}(X, \mathcal{D})}=\left(\|u\|_{L^{p}(X)}^{p}+\inf _{g \in \mathcal{D}(u)}\|g\|_{L^{p}(X)}^{p}\right)^{1 / p}, \quad u \in \operatorname{Lip}_{\beta}(X) .
$$

In order to formulate our results, we need a global version of the maximal function (4.1). To this end, we write $\mathcal{B}=\{B: B \subset X$ is a ball $\}$ and denote

$$
M_{\beta}^{\sharp, p} u(x)=M_{\beta, \mathcal{B}}^{\sharp, p} u(x)=\sup _{x \in B \in \mathcal{B}}\left(\frac{1}{\operatorname{diam}(B)^{\beta p}} f_{B}\left|u(y)-u_{B}\right|^{p} d \mu(y)\right)^{1 / p}, \quad x \in X,
$$

whenever $u: X \rightarrow \mathbb{R}$ is a $\beta$-Hölder function, i.e., $u \in \operatorname{Lip}_{\beta}(X)$.

By applying this global maximal function in Theorem 6.2 below, we provide a structure independent representation for the Sobolev norm that arises from an appropriate $\mathcal{D}$-structure; more specifically, we need to additionally assume that $\eta M_{\beta}^{\sharp, p} u \in \mathcal{D}(u)$ whenever $u$ is a $\beta$-Hölder function on $X$. Here $\eta$ is a constant that is independent of $u$. As we will see, this assumption holds in various applications.

Theorem 6.2. Suppose we are given a $\mathcal{D}$-structure $\mathcal{D}$ in a geodesic space $X$, with exponents $1<p<\infty$ and $0<\beta \leq 1$. Let $\eta>0$ and suppose that $\eta M_{\beta}^{\sharp, p} u \in \mathcal{D}(u)$ for every $u \in \operatorname{Lip}_{\beta}(X)$. Then there exists a constant $C=C\left(K_{p, p}, \beta, p, c_{\mu}, \eta\right) \geq 1$ such that

$$
C^{-1}\|u\|_{W_{\beta}^{p}(X, \mathcal{D})} \leq\left(\|u\|_{L^{p}(X)}^{p}+\left\|M_{\beta}^{\sharp, p} u\right\|_{L^{p}(X)}^{p}\right)^{1 / p} \leq C\|u\|_{W_{\beta}^{p}(X, \mathcal{D})}
$$

whenever $u \in \operatorname{Lip}_{\beta}(X)$.

Remark 6.4. Inequality (6.3) holds also when either one of the two quantities

$$
\|u\|_{W_{\beta}^{p}(X, \mathcal{D})}, \quad\left(\|u\|_{L^{p}(X)}^{p}+\left\|M_{\beta}^{\sharp, p} u\right\|_{L^{p}(X)}^{p}\right)^{1 / p}
$$

is infinite. In this case we can conclude that actually both of these quantities are infinite.

Proof of Theorem 6.2. The left inequality in (6.3) follows from the definitions and the assumption that $\eta M_{\beta}^{\sharp, p} u \in \mathcal{D}(u)$ for every $u \in \operatorname{Lip}_{\beta}(X)$. To prove the right inequality, we fix a point $x_{0} \in X$ and denote $B_{j}=B\left(x_{0}, j\right)$ and $\mathcal{B}_{j}=\left\{B=B(x, r): 2 B \subset B_{j}\right\}$ for $j \in \mathbb{N}$.

Fix $u \in \operatorname{Lip}_{\beta}(X)$ and $g \in \mathcal{D}(u)$. Observe that

$$
M_{\beta}^{\sharp, p} u(x)=\lim _{j \rightarrow \infty}\left(\mathbf{1}_{B_{j}}(x) M_{\beta, \mathcal{B}_{j}}^{\sharp, p} u(x)\right)
$$

\footnotetext{
${ }^{3}$ Conditions (D2) and (D3) imply the properties of a vector space and a norm; cf. [5, Theorem 1.5].
} 
whenever $x \in X$. Hence, by Fatou's lemma and Theorem 4.3, with $\varepsilon=0$, we obtain that

$$
\begin{aligned}
\int_{X}\left(M_{\beta}^{\sharp, p} u(x)\right)^{p} d \mu(x) & \leq \liminf _{j \rightarrow \infty} \int_{B_{j}}\left(M_{\beta, \mathcal{B}_{j}}^{\sharp, p} u(x)\right)^{p} d \mu(x) \\
& \leq C\left(K_{p, p}, \beta, p, c_{\mu}\right) \liminf _{j \rightarrow \infty} \int_{B_{j}} g(x)^{p} d \mu(x) \\
& \leq C\left(K_{p, p}, \beta, p, c_{\mu}\right) \int_{X} g(x)^{p} d \mu(x) .
\end{aligned}
$$

The right inequality in (6.3) follows by infimizing the above estimate over all $g \in \mathcal{D}(u)$.

\subsection{Universality of Hajłasz-Sobolev spaces}

By using Theorem 6.2, we shall now provide isomorphic representatives for the abstract Sobolev spaces in terms of certain Hajłasz-Sobolev spaces. This can be done as follows if $1<p<\infty$ and $0<\beta \leq 1$.

For each $\beta$-Hölder function $u: X \rightarrow \mathbb{R}$, we let $\mathcal{D}_{H}^{\beta, p}(u) \neq \emptyset$ be the family of all measurable functions $g: X \rightarrow[0, \infty]$ such that

$$
|u(x)-u(y)| \leq d(x, y)^{\beta}(g(x)+g(y))
$$

almost everywhere, i.e., there exists an exceptional set $N=N(g) \subset X$ for which $\mu(N)=0$ and inequality (6.5) holds for every $x, y \in X \backslash N$.

As we will see below, this construction gives a $\mathcal{D}$-structure

$$
\mathcal{D}_{H}^{\beta, p}=\left\{\mathcal{D}_{H}^{\beta, p}(u): u \in \operatorname{Lip}_{\beta}(X)\right\},
$$

with exponents $p$ and $\beta$, and with constants $K=2^{p}$ and $\tau=1$. The associated abstract Sobolev space is the so-called Hajlasz-Sobolev space that is denoted by

$$
M^{\beta, p}(X)=W_{\beta}^{p}\left(X, \mathcal{D}_{H}^{\beta, p}\right) .
$$

This space has been studied, e.g., in $[6,7,9]$. Our approach via completion is not standard. However, by the known density results of Hölder-functions [16, Proposition 4.5], the perhaps more conventional definition [16, pp. 194-195] yields an isomorphic Banach space.

Returning to the $\mathcal{D}$-structure conditions, it is straightforward to verify that condition (D1) is valid; in fact, even the stronger $(p, p)$-Poincaré inequality condition (D1') in Theorem 3.3 holds with a constant $K_{p, p}=2^{p}$; cf. [9, Theorem 5.15]. The two conditions (D2) and (D3) are also satisfied; we leave details to the reader. The validity of the last condition (D4) is a consequence of the following lemma.

Lemma 6.6. Let $1<p<\infty$ and $0<\beta \leq 1$, and fix a Borel set $E \subset X$. Let $u: X \rightarrow \mathbb{R}$ be a $\beta$-Hölder function and suppose that $v: X \rightarrow \mathbb{R}$ is such that $\left.v\right|_{X \backslash E}=\left.u\right|_{X \backslash E}$ and there exists a constant $\kappa \geq 0$ such that $|v(x)-v(y)| \leq \kappa d(x, y)^{\beta}$ for all $x, y \in X$. Then

$$
g_{v}=\kappa \mathbf{1}_{E}+g_{u} \mathbf{1}_{X \backslash E} \in \mathcal{D}_{H}^{\beta, p}(v)
$$

whenever $g_{u} \in \mathcal{D}_{H}^{\beta, p}(u)$.

Proof. Fix a function $g_{u} \in \mathcal{D}_{H}^{\beta, p}(u)$ and let $N \subset X$ be the exceptional set such that $\mu(N)=0$ and inequality (6.5) holds for every $x, y \in X \backslash N$ and with $g=g_{u}$.

Fix $x, y \in X \backslash N$. If $x, y \in X \backslash E$, then

$$
|v(x)-v(y)|=|u(x)-u(y)| \leq d(x, y)^{\beta}\left(g_{u}(x)+g_{u}(y)\right)=d(x, y)^{\beta}\left(g_{v}(x)+g_{v}(y)\right) .
$$

If $x \in E$ or $y \in E$, then

$$
|v(x)-v(y)| \leq \kappa d(x, y)^{\beta} \leq d(x, y)^{\beta}\left(g_{v}(x)+g_{v}(y)\right) .
$$

By combining the estimates above, we find that

$$
|v(x)-v(y)| \leq d(x, y)^{\beta}\left(g_{v}(x)+g_{v}(y)\right)
$$

whenever $x, y \in X \backslash N$. The desired conclusion $g_{v} \in \mathcal{D}_{H}^{\beta, p}(v)$ follows. 
The following corollary is a universality result for Hajłasz-Sobolev spaces $M^{\beta, p}(X)$. Namely, any abstract Sobolev space, rising from a suitable $\mathcal{D}$-structure, turns to be isomorphic to this particular Sobolev space $M^{\beta, p}(X)$.

Corollary 6.7. Suppose we are given a $\mathcal{D}$-structure $\mathcal{D}_{A}$ in a geodesic space $X$, with exponents $1<p<\infty$ and $0<\beta \leq 1$. Assume that there exists $\eta>0$ such that $\eta M_{\beta}^{\sharp, p} u \in \mathcal{D}_{A}(u)$ for every $u \in \operatorname{Lip}_{\beta}(X)$. Let $W_{p}^{\beta}(X)=W_{p}^{\beta}\left(X, \mathcal{D}_{A}\right)$. Then there exists $C \geq 1$ such that

$$
C^{-1}\|u\|_{W_{\beta}^{p}(X)} \leq\|u\|_{M^{\beta, p}(X)} \leq C\|u\|_{W_{\beta}^{p}(X)}
$$

whenever $u \in \operatorname{Lip}_{\beta}(X)$. Moreover, there exists a unique Banach-space isomorphism between the spaces $W_{\beta}^{p}(X)$ and $M^{\beta, p}(X)$ which is the identity on $W_{\beta}^{p}(X) \cap \operatorname{Lip}_{\beta}(X)=M^{\beta, p}(X) \cap \operatorname{Lip}_{\beta}(X)$.

Proof. By modifying the proof of inequality (4.13), it is straightforward to show that

$$
C\left(\beta, c_{\mu}\right) M_{\beta}^{\sharp, p} u \in \mathcal{D}_{H}^{\beta, p}(u), \quad u \in \operatorname{Lip}_{\beta}(X) .
$$

Applying Theorem 6.2 with the two $\mathcal{D}$-structures $\mathcal{D}_{A}$ and $\mathcal{D}_{H}^{\beta, p}$ yields the claim. Indeed, recall that by definitions $W_{p}^{\beta}(X)=W_{p}^{\beta}\left(X, \mathcal{D}_{A}\right)$ and $M^{\beta, p}(X)=W_{\beta}^{p}\left(X, \mathcal{D}_{H}^{\beta, p}\right)$.

\subsection{Universality of Newtonian spaces}

Fix $u \in \operatorname{Lip}(X)$ and $1<p<\infty$. Recall from $\S 5$ that $\mathcal{D}_{N}^{1, p}(u)$ is the family of all $p$-weak upper gradients $g \in L_{\text {loc }}^{p}(X)$ of the function $u$. Arguing as in $\S 5$, we find that

$$
\mathcal{D}_{N}^{1, p}=\left\{\mathcal{D}_{N}^{1, p}(u): u \in \operatorname{Lip}(X)\right\}
$$

is a $\mathcal{D}$-structure with exponents $\beta=1$ and $p$, if we assume that the $(1, p)$-Poincare inequality condition (D1) holds. The associated abstract Sobolev space is the so-called Newtonian space

$$
N^{1, p}(X)=W_{1}^{p}\left(X, \mathcal{D}_{N}^{1, p}\right)
$$

We remark that this notation is not entirely standard since Lipschitz functions need not be dense when the more conventional approach [14], [1, Definition 1.17] to the Newtonian space is adopted. However, when $X$ supports a $(1, p)$-Poincaré inequality (6.10) for all measurable functions instead of Lipschitz functions only, then the density result holds and the two definitions give isomorphic Banach spaces; c.f. [1, Theorem 5.1].

The following corollary extends and complements [14, Theorem 4.9] and [15, Theorem 4.3]. Observe that, by Corollary 6.7 and transitivity, it also produces a universality result for Newtonian spaces $N^{1, p}(X)$.

Corollary 6.9. Suppose that $X$ is a geodesic space and fix $1<p<\infty$. Suppose that there are constants $K>0$ and $\tau \geq 1$ such that the $(1, p)$-Poincaré inequality

$$
f_{B}\left|u(x)-u_{B}\right| d \mu(x) \leq K^{1 / p} \operatorname{diam}(B)\left(f_{\tau B} g(x)^{p} d \mu(x)\right)^{1 / p}
$$

holds whenever $B$ is a ball in $X$ and $g \in L_{\text {loc }}^{p}(X)$ is a p-weak upper gradient of $u \in \operatorname{Lip}(X)$. Then there exists a constant $C \geq 1$ such that

$$
C^{-1}\|u\|_{N^{1, p}(X)} \leq\|u\|_{M^{1, p}(X)} \leq C\|u\|_{N^{1, p}(X)}
$$

whenever $u \in \operatorname{Lip}(X)$. Moreover, there exists a unique Banach-space isomorphism between the spaces $M^{1, p}(X)$ and $N^{1, p}(X)$ which is the identity on $N^{1, p}(X) \cap \operatorname{Lip}(X)=M^{1, p}(X) \cap \operatorname{Lip}(X)$.

Proof. Arguing as in $\S 5$, we obtain a constant $C\left(1, c_{\mu}\right)>0$ such that $4 C\left(1, c_{\mu}\right) M_{1}^{\sharp, p} u \in L_{\text {loc }}^{p}(X)$ is a $p$-weak upper gradient of any given $u \in \operatorname{Lip}(X)$. The claim follows from Corollary 6.7. 


\section{REFERENCES}

[1] A. Björn and J. Björn. Nonlinear potential theory on metric spaces, volume 17 of EMS Tracts in Mathematics. European Mathematical Society (EMS), Zürich, 2011.

[2] S. Eriksson-Bique. Alternative proof of Keith-Zhong self-improvement. arXiv:1610.02129, 2016.

[3] J. García-Cuerva and J. L. Rubio de Francia. Weighted norm inequalities and related topics, volume 116 of North-Holland Mathematics Studies. North-Holland Publishing Co., Amsterdam, 1985.

[4] A. Gogatishvili, P. Koskela, and Y. Zhou. Characterizations of Besov and Triebel-Lizorkin spaces on metric measure spaces. Forum Math., 25(4):787-819, 2013.

[5] V. Gol'dshtein and M. Troyanov. Axiomatic theory of Sobolev spaces. Expo. Math., 19(4):289-336, 2001.

[6] P. Hajłasz. Sobolev spaces on metric-measure spaces. In Heat kernels and analysis on manifolds, graphs, and metric spaces (Paris, 2002), volume 338 of Contemp. Math., pages 173-218. Amer. Math. Soc., Providence, RI, 2003.

[7] P. Hajłasz and J. Kinnunen. Hölder quasicontinuity of Sobolev functions on metric spaces. Rev. Mat. Iberoamericana, 14(3):601-622, 1998.

[8] P. Hajłasz and P. Koskela. Sobolev meets Poincaré. C. R. Acad. Sci. Paris Sér. I Math., 320(10):1211-1215, 1995.

[9] J. Heinonen. Lectures on analysis on metric spaces. Universitext. Springer-Verlag, New York, 2001.

[10] J. Heinonen, P. Koskela, N. Shanmugalingam, and J. T. Tyson. Sobolev spaces on metric measure spaces: An approach based on upper gradients, volume 27 of New Mathematical Monographs. Cambridge University Press, Cambridge, 2015.

[11] R. Jiang, N. Shanmugalingam, D. Yang, and W. Yuan. Hajłasz gradients are upper gradients. J. Math. Anal. Appl., 422(1):397-407, 2015.

[12] S. Keith and X. Zhong. The Poincaré inequality is an open ended condition. Ann. of Math. (2), 167(2):575599, 2008.

[13] E. Kreyszig. Introductory functional analysis with applications. Wiley Classics Library. John Wiley \& Sons, Inc., New York, 1989.

[14] N. Shanmugalingam. Newtonian spaces: an extension of Sobolev spaces to metric measure spaces. Rev. Mat. Iberoamericana, 16(2):243-279, 2000.

[15] N. Shanmugalingam. A universality property of Sobolev spaces in metric measure spaces. In Sobolev spaces in mathematics. I, volume 8 of Int. Math. Ser. (N. Y.), pages 345-359. Springer, New York, 2009.

[16] N. Shanmugalingam, D. Yang, and W. Yuan. Newton-Besov spaces and Newton-Triebel-Lizorkin spaces on metric measure spaces. Positivity, 19(2):177-220, 2015.

(J.K.) Department of Mathematics, Aalto University, P.O. Box 11100, Fi-00076 Aalto UniVERSITY, FINLAND

E-mail address: juha.k.kinnunen@aalto.fi

(J.L.) University of Jyvaskyla, Department of Mathematics and Statistics, P.O. Box 35, Fi40014 University of JyVASKYla, Finland

E-mail address: juha.lehrback@jyu.fi

(A.V.V.) University of Jyvaskyla, Department of Mathematics and Statistics, P.O. Box 35, FI-40014 UNIVERSity OF JYVASKYLA, Finland

E-mail address: antti.vahakangas@iki.fi

(X.Z.) Department of Mathematics and Statistics, Gustaf Hällströmin katu 2B, FI-00014 UniVERSITY OF HELSINKI, FINLAND

E-mail address: xiao.x.zhong@helsinki.fi 